PNNL-11001

UC-810

Project Technical Information

RECEIVED

MAR 221996

OSTI

Strategy for Addressing Composition

Uncertainties in a Hanford High-

Level Waste Vitrification Plant

M.F. Bryan

G.F. Piepel

March 1996

Prepared for the U.S. Department of Energy under Contract DE-AC06-76RLO 1830

Pacific Northwest National Laboratory

Operated for the U.S. Department of Energy by Battelle Memorial Institute 
6 
PNNL-11001

UC-810

Project Technical Information

\title{
Strategy for Addressing Composition Uncertainties in a Hanford High-Level Waste Vitrification Plant
}

\author{
M. F. Bryan \\ G. F. Piepel
}

March 1996

Prepared for

the U.S. Department of Energy

under Contract DE-AC06-76RLO 1830

Pacific Northwest National Laboratory

Richland, Washington 99352

Reprint of historical document PVTD-C94-02.0411 Rev. 0, dated December 1994. Data, formatting, and other conventions reflect standards at the origimal dare of printing. Technical peer reviews and editorial reviews may not have been performed. 


\title{
DISCLAIMER
}

This report was prepared as an account of work sponsored by an agency of the United States Government. Neither the United States Government nor any agency thereof, nor Battelle Memorial Institute, nor any of their employees, makes any warranty, express or implied, or assumes any legal liability or responsibility for the accuracy, completeness, or.usefulness of any information, apparatus, product, or process disclosed, or represents that its use would not infringe privately owned rights. Reference herein to any specific commercial product, process, or service by trade name, trademark, manufacturer, or otherwise does not necessarily constitute or imply its endorsement, recommendation, or favoring by the United States Government or any agency thereof, or Battelle Memorial Institute. The views and opinions of authors expressed herein do not necessarily state or reflect those of the United States Government or any agency thereof.

\author{
PACIFIC NORTHWEST NATIONAL LABORATORY \\ operated by \\ BATTELLE \\ for the \\ UNITED STATES DEPARTMENT OF ENERGY \\ under Contract DE-ACO6-76RLO 1830
}

Printed in the United States of America

Available to DOE and DOE contractors from the

Office of Scientific and Technical information, P.O. Box 62, Oak Ridge, TN 37831; prices, available from (615) 576-8401.

Available to the public from the National Technical Information Service, U.S. Department of Commerce, 5285 Port Royal Rd., Springrield, VA 22161 


\section{SUMMARY}

Various requirements will be imposed on the feed material and glass produced by the high-level waste (HLW) vitrification plant at the Hanford Site. A statistical process/product control system will be used to control the melter feed composition and to check and document product quality. Two general types of uncertainty are important in HLW vitrification process/product control: model uncertainty and composition uncertainty. Model uncertainty is discussed by Hrma, Piepel, et al. (1994). Composition uncertainty includes the uncertainties inherent in estimates of feed composition and other process measurements. Because feed composition is a multivariate quantity, multivariate estimates of composition uncertainty (i.e., covariance matrices) are required.

Three components of composition uncertainty will play a role in estimating and checking batch and glass attributes: batch-to-batch variability, within-batch uncertainty, and analytical uncertainty. This document reviews the techniques to be used in estimating and updating composition uncertainties and in combining these composition uncertainties with model uncertainty to yield estimates of (univariate) uncertainties associated with estimates of batch and glass properties.

Although data accumulated during HLW vitrification operations will be used to estimate and update composition uncertainties, uncertainty estimates are required for developing, testing, and initializing the process/product control system. Therefore, it is desirable to obtain data from similar vitrification operations, such as the Defense Waste Processing Facility (DWPF) at the Savannah River Site and the West Valley Demonstration Project (WVDP). This document identifies the type, extent, and possible sources of data required for estimating uncertainties. It is recommended that all composition variance and covariance estimates be based on samples of size 20 . Due to the hierarchical nature of the variance and covariance components, the smallest set of data satisfying this condition for each component of uncertainty would be one with 80 observations: 20 batches, with two samples drawn from each batch, and two analyses performed for each sample. Different allocations of sampling effort are possible. Of course, the larger the data set, the better the resulting uncertainty estimates. 


\section{GLOSSARY}

Acceptable--A batch or composition for which all applicable requirements will be met (with some degree of statistical confidence, as discussed in the body of the document).

Accuracy--Closeness of agreement between an estimated or measured value of a quantity and the true value (or an accepted reference value) of the quantity.

Analytical uncertainty--Uncertainty among analytical results from the same sample. This is a composite form of uncertainty, made up of variability induced during sample preparation and the inherent error of the measurement process itself.

Batch--A discrete quantity of material (waste, frit, recycle, or a combination of the three) to be processed by the Hanford high-level waste (HLW) vitrification plant.

Batch-to-batch variabilitv--Heterogeneity between batches made from the same waste type.

Bias--Consistent departures of measured or estimated quantities from the true value (see also error).

Components of covariance--Covariance matrices representing hierarchical levels of uncertainty for multivariate data.

Components of variance--Variances representing hierarchical levels of uncertainty in univariate data.

Composition--The proportions of each chemical species in a batch of material to be processed by the HLW vitrification plant; usually expressed as mass fractions of nine major oxides $\left(\mathrm{SiO}_{2}, \mathrm{~B}_{2} \mathrm{O}_{3}, \mathrm{Na}_{2} \mathrm{O}, \mathrm{Li}_{2} \mathrm{O}, \mathrm{CaO}, \mathrm{MgO}, \mathrm{Fe}_{2} \mathrm{O}_{3}, \mathrm{Al}_{2} \mathrm{O}_{3}, \mathrm{ZrO}_{2}\right)$ and a catchall tenth category, Others. In some cases, individual species normally included in "Others" may be segregated.

Composition uncertainty--Uncertainty in measured or estimated quantities stemming - from variability in material and/or sampling and analytical error.

Compositional data--A type of multivariate data in which the numerical values in each datum are the proportions (or percentages) of the individual components of the material or characteristic being represented by the datum. From their nature as proportions (percentages), these numerical values must lie between 0 and 1 ( 0 and 100\%), inclusive, and they must sum to 1 (100\%). 
Confidence--A measure of the long-run performance of a statistical procedure, expressed as the probability that the procedure produces the advertised result. For example, the procedure for producing a $95 \%$ confidence interval for the mean of a population has a 95\% chance of producing an interval that traps the mean. Note that confidence pertains to the procedure and not to any particular result.

Confidence interval--A type of statistical interval designed to trap, with specified confidence, a single fixed true value, such as the mean of a random variable.

Correlation--A standardized covariance which must lie between -1 and 1. Correlation is computed by dividing the covariance between two random variables by the product of the standard deviations of the two variables.

Correlation matrix--A standardized representation of the interrelationships between individual quantities that make up a multivariate datum. The correlation matrix is a symmetric matrix with 1's on the diagonal and the pairwise correlations in the offdiagonal positions.

Covariance-A measure of the tendency of two random quantities to vary together. Covariance is defined as the expected value of the product of the deviations of the two random quantities from their respective means, i.e., Covariance $(X, Y)=E\left(X-\mu_{X}\right)(Y-$ $\left.\mu_{Y}\right)$. Positive covariance indicates that the two quantities tend to increase or decrease together. Negative covariance indicates that one quantity tends to increase while the other decreases (or vice versa). Covariance can be estimated from a sample of n pairs $\left(X_{i}, Y_{i}\right), i=1, \ldots, n$, with the formula

$$
\operatorname{Cov}(X, Y)=\frac{1}{n-1} \sum_{I=1}^{n}\left(X_{i}-X\right)\left(Y_{i}-Y\right) .
$$

Covariance components--See components of covariance.

Covariance matrix-A representation of the uncertainties and interrelationships between individual quantities that make up a multivariate datum. The covariance matrix is a symmetric matrix with the variances of the individual quantities on the diagonal and the pairwise covariances in the off-diagonal positions.

Direct constraints--Requirements and constraints on HLW material (feed composition, melt, and glass) that pertain directly to measured quantities (e.g., oxide mass fractions) or to known functions of these measured quantities.

E(•)-See expected value. 
Error--The random deviation of a measured or estimated quantity from the true value, related to the imperfection of the sampling or analytical procedure.

Expectation--See expected value.

Expected value--The average value of a random quantity; in general, given a function, $h(X)$, of a random variable $X$, the expected value (or expectation) of $h(X)$ is defined as

$$
E(h(x)) \equiv \int_{-\infty}^{\infty} h(x) d F(x)=\int_{-\infty}^{\infty} h(x) f(x) d x .
$$

Feed--A generic term used to refer to any material being processed in the HLW vitrification plant, upstream of the melter itself (see also melt).

Long-term variability--Heterogeneity in material over waste types.

Mean-A statistical measure of the average or central tendency of a random quantity. The mean, $\mu$, of a random variable $\mathrm{X}$ is simply the expected value of $\mathrm{X}$, i.e., $\mu=E(\mathrm{X})$. The mean can be estimated from a sample, $X_{i}, i=1, \ldots, n$, with the formula

$$
\bar{x}=\frac{1}{n} \sum_{i=1}^{n} x_{i} \text {. }
$$

Melt--Material being processed by the HLW vitrification plant in the melter before it has cooled and solidified into glass. Before reaching the melter, this material will be referred to as feed.

Model uncertaintv--Uncertainty in an estimated property value stemming from imperfection of the model used to relate feed composition to the property.

Modelled properties--Properties of feed, melt, or glass for which statistical models are being developed to relate feed composition to the property values.

Moments--The expected values of powers of a random variable, $\mathrm{X}$. The first moment, $E(X)$, is the mean, $\mu$. Central moments are expected values of powers of the difference between $X$ and its mean; the second central moment, $E(X-\mu)^{2}$, is the variance.

Multiple-batch requirement or constraint--A requirement or constraint imposed over a set of batches to be processed by the HLW vitrification plant (e.g., a property for which the requirement is imposed on an entire waste type, rather than on the individual batches constituting the waste type). See also single-batch requirement or constraint. 
Prediction interval--A type of statistical interval designed to trap, with specified confidence, a single random true value, such as a new observation of a random variable.

Relative standard deviation--The ratio of the standard deviation to the mean, estimated by $S / \mathrm{X}$.

S--See standard deviation.

$\underline{S^{2}-\text {-See variance. }}$

Sampling uncertainty--See within-batch uncertainty.

Single-batch requirement or constraint--A requirement or constraint imposed on each individual batch to be processed by the HLW vitrification plant, with no reference to the characteristics of preceding or succeeding batches. See also multiple-batch requirement or constraint.

Standard deviation--Defined as the square root of the variance, the standard deviation is a measure of uncertainty on the same scale as the original quantity. Roughly, the standard deviation is the average distance of an observed value from the mean.

Tolerance interval--A statistical procedure designed to trap, with specified confidence, a specified proportion of the distribution of a random variable. The proportion of the distribution to be trapped is termed the content of the tolerance interval. For example, a $95 \% / 99 \%$ tolerance interval traps $99 \%$ of the distribution with $95 \%$ confidence.

Uncertainty-A general term used to refer to any of several measures of the random behavior of some quantity; for example, see composition uncertainty, model uncertainty, variability, and error.

Variability--Uncertainty related to heterogeneity in material under examination; for example, see batch-to-batch variability.

Variance--A statistical measure of the random behavior of some quantity. Variance is defined as the expected value of the squared deviation of a random variable, $\mathrm{X}$, from its mean, $\mu$, i.e., Variance $(\mathrm{X})=E(\mathrm{X}-\mu)^{2}$. Variance can be estimated from a sample, $\mathrm{X}_{\mathrm{i}}$, $\mathrm{i}=1, \ldots, \mathrm{n}$, with the formula

$$
s^{2}=\frac{1}{n-1} \sum_{i=1}^{n}\left(x_{i}-x\right)^{2}
$$

Variance components--See components of variance. 
Variance-covariance matrix--See covariance matrix.

WAPS properties and requirements--Properties of and requirements on glass produced by the HLW vitrification plant, as detailed in the Waste Acceptance Product Specifications (USDOE 1993). These properties and requirements are related to the performance of the glass in the repository.

Waste type--A relatively homogeneous stream of waste to be processed by the HLW vitrification plant. Several to many batches will be made from a single waste stream.

Within-batch uncertainty--Uncertainty among samples from the same process batch; this is a composite form of uncertainty, made up of variability (heterogeneity) in the process batch and the inherent error of the sampling process itself.

$\underline{X}-$-See mean. 
ANOVA--Analysis of Variance

CVS--Composition Variation Study

DWPF--Defense Waste Processing Facility

HLW--High-Level Waste

IID--Independent and identically distributed

PCCS--Product Composition Control System

PCT--Product Consistency Test

PNL--Pacific Northwest Laboratory

PVTD--PNL Vitrification Technology Development

PPMD--Process/Product Model Development

RSD--Relative standard deviation

WAPS--Waste Acceptance Product Specifications

WCP.-Waste Form Compliance Plan

WQR--Waste Form Qualification Report

WVDP--West Valley Demonstration Project 


\section{CONTENTS}

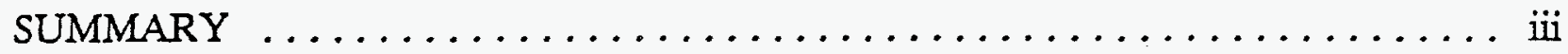

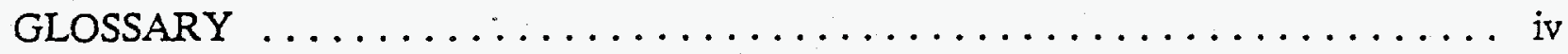

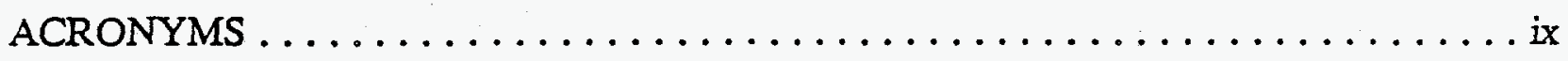

1.0 INTRODUCTION $\ldots \ldots \ldots \ldots \ldots \ldots \ldots \ldots \ldots \ldots \ldots \ldots \ldots \ldots \ldots \ldots$

2.0 ESTIMATING AND MANIPULATING UNCERTAINTIES $\ldots \ldots \ldots \ldots \ldots .5$

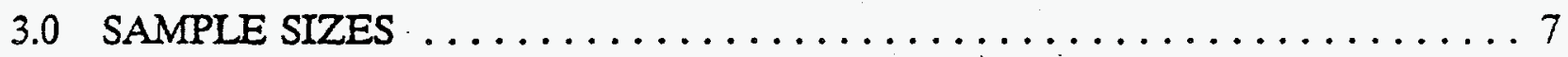

4.0 POSSIBLE SOURCES OF DATA $\ldots \ldots \ldots \ldots \ldots \ldots \ldots \ldots \ldots \ldots \ldots$

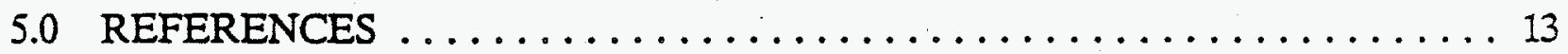

APPENDIX A - STATISTICAL BACKGROUND ............. A.1

A.1 DISTRIBUTION AND DENSITY FUNCTIONS $\ldots \ldots \ldots \ldots \ldots$ A.2

A.2 MEAN AND VARIANCE $\ldots \ldots \ldots \ldots \ldots \ldots \ldots \ldots \ldots \ldots$ A.4

A.3 MULTIVARIATE DATA, COVARIANCE, AND CORRELATION . . A. 6

A.4 ESTIMATING POPULATION PARAMETERS WITH SAMPLE STATISTICS $\ldots \ldots \ldots \ldots \ldots \ldots \ldots \ldots \ldots$ A.9

A.5 PROPERTY MODEL NOTATION . ............... A.13

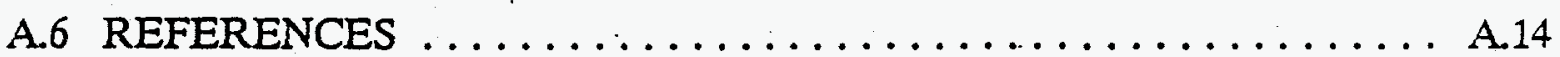

APPENDIX B - ESTIMATING AND MANIPULATING UNCERTAINTIES . . . B.1

B.1 THE MODEL FOR COMPOSITION UNCERTAINTY IN HLW VITRIFICATION DATA $\ldots \ldots \ldots \ldots \ldots \ldots \ldots \ldots . .2$

B.2 THE BATCH MEAN AND ASSOCIATED UNCERTAINTIES $\ldots \ldots$ B.3

B.3 THE MULTIPLE-BATCH MEAN AND ASSOCIATED UNCERTAINTIES $\ldots \ldots \ldots \ldots \ldots \ldots \ldots \ldots \ldots \ldots \ldots \ldots \ldots \ldots \ldots$

B.4 ESTIMATING VARIANCE COMPONENTS $\ldots \ldots \ldots \ldots \ldots \ldots \ldots$ B.8 
B.5 PROPAGATING UNCERTAINTIES $\ldots \ldots \ldots \ldots \ldots \ldots \ldots$ B. 13

B.6 COMBINING UNCERTAINTIES $\ldots \ldots \ldots \ldots \ldots \ldots \ldots \ldots$ B.16

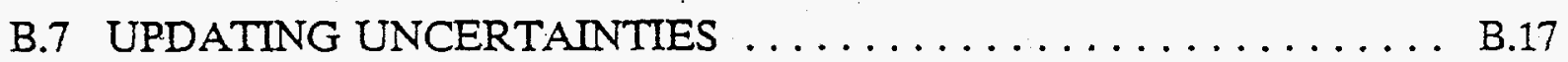

B.8 REFERENCES $\ldots \ldots \ldots \ldots \ldots \ldots \ldots \ldots \ldots \ldots \ldots \ldots \ldots \ldots$ B.20 


\subsection{INTRODUCTION}

A high-level waste (HLW) vitrification plant at the Hanford Site is planned to immobilize transuranic and high-level radioactive waste in borosilicate glass. Each batch of plant feed material will have to meet certain requirements related to plant performance, and the resulting glass must meet requirements imposed by the Waste Acceptance Product Specifications (WAPS, USDOE 1993). Similar vitrification operations will be performed in the Defense Waste Processing Facility (DWPF) at the Savannah River Site. DWPF has developed a Product Composition Control System (PCCS) for controlling melter feed composition and for checking and documenting product quality (Postles and Brown 1991, WSRC 1993). The HWVP Project Waste Form Qualification Program Plan (Randklev 1993) calls for the development of a product composition control-type system to perform these functions for the Hanford HIW vitrification plant.

The objectives of the Process/Product Model Development (PPMD) cost account of the Pacific Northwest Laboratory Vitrification Technology Development (PVTD) Project includes developing and testing methods and algorithms for the Hanford HLW vitrification process/product control system. Various aspects of these methods and algorithms are discussed by Bryan and Piepel (1993), Bryan and Piepel (1994), and Bryan, Piepel, and Simpson (1994a and 1994b). Due to uncertainties in the data and models to be used in controlling HLW vitrification operations and product quality, these methods and algorithms must be statistical in nature. For each process batch, the algorithms will: 1) choose a target melter feed composition, 2) estimate the actual melter feed composition by reconciling various process measurements, 3) use the estimated melter feed composition to estimate, check, and document various batch and product characteristics, and 4) recommend remediation strategies for process batches that do not meet requirements. 
Attributes $^{(a)}$ of a process batch and the resulting glass are largely determined by the composition of the feed material. In addition, remediation options are limited once material reaches the melter. Therefore, the relationships between "upstream" feed composition and "downstream" batch and glass properties will be exploited to ensure acceptable batch and glass properties and to perform any required remediation before material enters the melter.

Development of empirical models relating melter feed composition to important batch and glass properties is one objective of the ongoing Composition Variation Study (CVS) (Hrma, Piepel, et al. 1994). ${ }^{\text {(b) }}$ The Hanford HLW process/product control system will use these models to estimate batch and glass properties as functions of feed composition.

Two general types of uncertainty are important in HLW vitrification process/product control: model uncertainty and composition uncertainty. ${ }^{(c)}$ Model uncertainty derives from the use of empirical models to calculate batch and glass properties. This uncertainty must be taken into account when estimating and checking modelled properties. Estimating this type of uncertainty is another objective of the CVS and therefore is not discussed in this document.

(a) Established usage reserves the word property for characteristics of the melt and glass (which will usually be estimated via models based on feed composition), but requirements and constraints will also be imposed on feed composition (oxide mass fractions and functions thereof). To avoid confusion, the word attribute is used here to refer to any characteristic of HLW vitrification material (feed, melt, and glass).

(b) The form of these empirical models is given in Section A.5 of Appendix A. In most cases, the CVS models actually relate feed composition to the natural logarithm of property value.

(c) Composition uncertainty might also be called data uncertainty, since it exists to some degree in virtually any process used to collect data. However, the main type of data to be used in HLW vitrification process/product control will be compositional data, so the more specific term is used here. This term should be understood to include uncertainties in other types of data (e.g., tank level measurements) employed in HLW vitrification process/product control. 
Composition uncertainty is the uncertainty inherent in estimates of feed composition. This type of uncertainty may stem from heterogeneity in material, imperfection of measurement processes, or both. Composition uncertainty must be taken into account when estimating and checking any batch or glass attribute. Three components of composition uncertainty will play a role in estimating and checking batch and glass attributes:

- Batch-to-batch variability - Heterogeneity between process batches made from the same waste type and frit. This type of heterogeneity might also be called between-batch variability or within-waste type variability.

- Within-batch uncertainty - A combination of heterogeneity within a single process batch and any imperfections in the sampling process. This type of uncertainty might also be called sampling uncertainty.

- Analytical uncertainty - A combination of heterogeneity within a sample, variability induced during sample preparation, and any imperfections in the analytical process.

Data accumulated during HLW vitrification operations will be used to estimate and update these composition uncertainties. However, in order to develop and test the HLW vitrification process/product control system, estimates of these various uncertainties must be available. In addition, during the early phases of vitrification operations, the data available may be insufficient to adequately estimate these uncertainties. Therefore, other sources of uncertainty estimates must be found. Although it may be possible to construct reasonable uncertainty estimates from process knowledge (e.g., via simulation), a more desirable approach would be to use uncertainty estimates from vitrification operations similar to those to be employed at Hanford. The obvious candidates are the DWPF and the vitrification operations to be carried out in the West Valley Demonstration Project (WVDP). In fact, the main purpose of this document is to facilitate acquisition of data from other vitrification projects by briefly explaining the methods to be used in Hanford's HLW vitrification process/product control system and outlining the types of data required to test and initialize this system. Accordingly, the 
main topics addressed by this documient are

- briefly reviewing the techniques to be used to estimate and manipulate composition uncertainties (Section 2);

- establishing the type and extent of data required for estimating uncertainties (Section 3); and

- identifying possible sources of such information (Section 4).

Basic statistical notation, concepts, and techniques appear in Appendix A. Technical details of estimating and manipulating uncertainties appear in Appendix B. 


\subsection{ESTIMATING AND MANIPULATING UNCERTAINTIES}

The HLW vitrification process/product control system will use confidence and tolerance intervals to identify acceptable feed compositions and to check the various requirements imposed on the feed material. Lower and upper confidence or tolerance limits will be compared to lower and upper limits on attribute values. The proper use of confidence and tolerance intervals is discussed in more detail by Bryan and Piepel (1994) and Bryan, Piepel, and Simpson (1994a). Three points are important for this discussion: 1) the confidence or tolerance intervals will be constructed on an attribute-by-attribute basis, i.e., univariate confidence/tolerance intervals will be employed, 2) inferences are required for two types of means (batch means and multiple-batch, or waste type, means), and 3) each confidence/tolerance interval requires an estimate of the uncertainty about the estimated attribute value. ${ }^{(a)}$ These points give rise to technical difficulties, which are discussed briefly below. Technical details of the procedures appear in Appendix B.

As mentioned in Section 1, many glass property values will be estimated from empirically derived models relating feed composition to the resulting property values. Other attributes may be expressed as functions of feed composition. Feed composition will usually be expressed as mass fractions of nine individual oxides $\left(\mathrm{SiO}_{2}, \mathrm{~B}_{2} \mathrm{O}_{3}, \mathrm{Na}_{2} \mathrm{O}\right.$, $\mathrm{Li}_{2} \mathrm{O}, \mathrm{CaO}, \mathrm{MgO}, \mathrm{Fe}_{2} \mathrm{O}_{3}, \mathrm{Al}_{2} \mathrm{O}_{3}$, and $\mathrm{ZrO}_{2}$ ) and a catchall tenth category, Others. Since these mass fractions must sum to one, ${ }^{(b)}$ they are obviously not independent of one another; hence their simultaneous behavior is of interest. Thus, feed composition is a

(a) In HLW vitrification process/product control, confidence intervals will be used to draw inference about the means of the attributes of interest and therefore require estimates of the standard deviations of the sample means (often called standard errors). Tolerance intervals will be used to draw inference about some proportion (or percentile) of the distribution of attribute values and therefore require estimates of the standard deviation of the underlying distribution of attribute values in the batches under consideration.

(b) This restriction is the defining feature of compositional data, which is discussed in detail by Aitchison (1986). 
multivariate quantity and requires a multivariate estimate of uncertainty (i.e., a covariance matrix). On the other hand, each inference pertains to a single attribute, and therefore requires a univariate estimate of the uncertainty associated with this attribute. Therefore, methods are required for combining multivariate estimates of composition uncertainty to yield a univariate uncertainty estimate for each attribute. The HLW vitrification process/product control system will use error propagation (Section B.5 of Appendix B) to derive these univariate uncertainty estimates for each attribute of interest.

As discussed in Section 1, each measured or estimate quantity is subject to at least three sources of uncertainty: batch-to-batch variability, within-batch uncertainty, and analytical uncertainty. Proper estimation of the uncertainty associated with batch means and multiple-batch means requires proper combination of these separate, hierarchically structured variance components. ${ }^{\text {(a) }}$ The uncertainties associated with batch means and multiple-batch means are discussed in Appendix B Sections B.2 and B.3, respectively. Methods for estimating and combining variance components (both univariate and multivariate) are discussed in Appendix B Sections B.4 and B.6, respectively. (b) It will also be necessary to combine composition and model uncertainties; the method discussed in Section B.6 of Appendix B will be used here as well. Finally, as data accumulate during HLW vitrification operations, it will be necessary to update uncertainty estimates. One possible method for updating uncertainty estimates appears in Section B.7 of Appendix B. Other methods are discussed by Adams (1994) and by Bryan, Piepel, and Simpson (1994b).

(a) Once available, estimates of variance components will also be useful in allocating sampling effort (Cochran 1977) and in process monitoring.

(b) Estimating variance components is a complicated process, heavily dependent upon the type and extent of available data. More complete treatment of variance component estimation is given by Searle, Casella, and McCulloch (1992). 


\subsection{SAMPLE SIZES}

The sample size required for estimating a variance (or its square root, the standard deviation) is relatively easy to calculate when the underlying population follows a normal distribution. In this case, the usual estimator of variance, $S^{2}$, follows a multiple of a chi-square distribution with $\mathrm{n}-1$ degrees of freedom (Lindgren, 1976, p. 334, Theorem 4); specifically,

$$
\frac{(n-1) S^{2}}{\sigma^{2}}-\chi^{2}(n-1)
$$

This fact can be used to compute sample sizes (i.e., the number of samples) required for estimating a variance or standard deviation with specified accuracy. (a) Table 1 presents the sample sizes $(n)$ required for estimating a standard deviation with specified levels of precision and confidence. For example, to estimate a standard deviation with $20 \%$ precision and $90 \%$ confidence, a sample of size 20 is required.

In a multivariate situation, interest lies in an entire covariance matrix, not just in a single variance. When feed composition is estimated in terms of nine oxide mass fractions and Others, 10 variances and 45 covariances $^{(b)}$ must be estimated. Although the sample size calculations above hold for each variance (and, as indicated by

(a) The method used here for estimating sample size is detailed by Bryan, Piepel, and Simpson (1994b). This method is an adaptation of methods described by Hahn and Meeker (1991, pp. 141-144).

(b) Although a $10 \times 10$ matrix has 100 elements, the symmetric nature of covariance and correlation matrices reduces the number of distinct elements that must be estimated to 45 . In fact, as long as the measured compositions sum to one, only 35 covariances must be estimated -- the remaining 10 covariances can be estimated from the fact that any row or column of the covariance matrix must sum to zero. This. is related to the inherent singularity of the covariance matrix for compositional data (see Aitchison 1986). However, this technical point is of little importance here. 
TABLE 1. Sample Sizes Required for Estimating Standard Deviation with Specified Levels of Precision and Confidence

\begin{tabular}{|c|c|c|c|c|}
\hline \multirow{2}{*}{ Precision } & \multicolumn{4}{|c|}{ Sample Sizes Required for Confidence = } \\
\cline { 2 - 5 } & $\mathbf{8 0 \%}$ & $\mathbf{9 0 \%}$ & $\mathbf{9 5 \%}$ & $\mathbf{9 9 \%}$ \\
\hline $1 \%$ & 3488 & 8174 & 13507 & 27084 \\
\hline $5 \%$ & 132 & 322 & 538 & 1088 \\
\hline $10 \%$ & 31 & 79 & 134 & 274 \\
\hline $20 \%$ & 7 & 20 & 34 & 70 \\
\hline $30 \%$ & 2 & 9 & 16 & 32 \\
\hline $40 \%$ & 2 & 5 & 9 & 19 \\
\hline $50 \%$ & 2 & 4 & 6 & 12 \\
\hline
\end{tabular}

simulation results, each covariance) individually, the problem of simultaneous inference arises in estimating the entire composition covariance matrix. When desired levels of precision and confidence must be obtained for several quantities simultaneously, the sample size required is larger than that required for a single quantity, and this required sample size increases rapidly as the number of estimated quantities increases. Simulation results presented by Bryan, Piepel, and Simpson (1994b) indicate that reasonably precise simultaneous estimation of a single $10 \times 10$ composition covariance matrix requires a very large sample (on the order of hundreds of observations). This problem is aggravated by the need to estimate composition covariance components (i.e., several $10 \times 10$ covariance matrices) for HLW vitrification process/product control.

Fortunately, in HLW vitrification process/product control, interest lies not in the multivariate composition uncertainties (covariance matrices and components of covariance) per se, but only in their effects on the uncertainties in estimated attribute values. As part of the simulation study mentioned above, each empirical composition covariance matrix was propagated through five first-order CVS property models (the 
models for viscosity and electrical conductivity at $1150^{\circ} \mathrm{C}$, and the models for Product Consistency Test (PCT) results for boron, lithium, and sodium). ${ }^{\text {(a) }}$ The univariate relative precision (as measured by the RSD) of each property improves much more quickly with increasing sample size than does that associated with multivariate composition uncertainty estimation. ${ }^{(b)}$ Even with a sample size of only 10 compositions to estimate a composition covariance matrix, the individual univariate property uncertainty estimates have relative precisions of about $23 \%$. Thus, even though fairly small samples yield relatively poor estimates of composition covariance matrices, these covariance matrices (when propagated through property models) yield relatively good estimates of univariate property uncertainties.

For adequate precision and confidence in estimates of property uncertainties due to composition uncertainties, it is recommended that composition variance and covariance estimates be based on samples of size 20 (which, according to Table 1, corresponds to a $90 \%$ confidence and $20 \%$ precision in univariate uncertainty estimation) or greater. Estimation of variance (and covariance) components is usually based on observations replicated at each of the levels of the variance hierarchy. In the HLW vitrification process, there are three levels in this variance hierarchy (and hence three variance components): batch-to-batch variability, within-batch uncertainty, and analytical

(a) As mentioned in Section 1, the CVS models actually predict the natural logarithm of these properties. Since the standard deviation of $\ln (\mathrm{Y})$ can be shown to be approximately equal to the relative standard deviation (RSD) of $Y$, error propagation using models that predict $\ln$ (property) yields estimates of RSDs on the original property scales.

(b) Basic statistical theory for simple estimation problems suggests that the standard deviation of an estimator should decrease (i.e., precision should increase) at a rate proportional to the square root of sample size. In other words, the standard deviation of an estimator based on $n_{1}$ samples should be approximately $\sqrt{ }\left(n_{2} / n_{1}\right)$ times the standard deviation of an estimator based on $n_{2}$ samples. This pattern appeared in the simulation results. 
uncertainty. (a) Estimates of each of these uncertainties should be based on samples of size 20 or greater. For example, the smallest set of data satisfying this condition for each component of uncertainty would be one with 80 observations: 20 batches, with two samples drawn from each batch, and two analyses (estimates of feed composition) performed for each sample. (b) Different allocations of sampling effort are possible. (especially if it is decided that some precision can be sacrificed in estimation of batch-tobatch variability). Of course, the larger the data set, the better the resulting uncertainty estimates.

(a) Each attribute measured as part of the HLW, vitrification process will be modelled with a two-way nested random model. This model and the associated sample sizes and degrees of freedom are discussed in Sections B.1 and B.4 of Appendix B, and by Searle, Casella, and McCulloch (1992).

(b) Because of the nested structure of the three main sources of composition variation in the recommended $20 \times 2 \times 2$ data set, two samples per batch for each of 20 batches will yield 20 degrees of freedom for estimating within-batch uncertainty, and two analyses per sample will yield 40 degrees of freedom for estimating analytical uncertainty. 


\subsection{POSSIBLE SOURCES OF DATA}

Previous statistical investigations performed as part of PVTD work have used several sources of data on composition uncertainties, e.g., the Corning RR6 and other data sets described by Anderson and Piepel (1993), and an analytical covariance. matrix constructed from simulation of a hypothetical analytical process (Bryan, Piepel, and Simpson 1994b). However, none of these data sources has been completely satisfactory. Each has been inadequate for proper uncertainty estimation, not clearly tied to the sampling and analytical processes to be used in the Hanford HLW vitrification process, or both. Data meeting both of these criteria will be required in developing and testing the Hanford HLW vitrification process/product control system. Such data should become available during design, testing and construction of the Hanford HLW vitrification plant. However, in the interim, it is desirable to obtain reasonable "substitute" data to be used in developing and testing the Hanford HLW vitrification process/product control system.

The obvious sources of such "substitute" data are the data generated during development and testing of the Savannah River and West Valley vitrification processes (DWPF and WVDP, respectively). DWPF has generated bench and prototypic test data from which composition covariance matrices and variance components have been estimated (Reeve 1992a, 1992b, and 1993). Unfortunately, the data from these studies were not included in Reeve's reports, so the data would have to be obtained from DWPF for use at Hanford. Data from the DWPF Startup Test Program (including Waste Form Qualification Runs) and a statistical measurement error model similar to that discussed in Section B.1 of Appendix B will be used to estimate variance components related to within-canister variation in glass properties (Edwards 1994). Much of these data could be valuable in providing initial estimates of at least withinbatch and analytical uncertainties expected in the Hanford HLW vitrification process (since the sampling and analytical procesșes should be similar). The batch-to-batch variance component depends on heterogeneity of the feed stream. Therefore, some 
information on the relative heterogeneity of the DWPF and Hanford feed streams must be used to judge the value of DWPF's batch-to-batch covariance (matrix) component as an initial estimate of the batch-to-batch variability in the Hanford HLW vitrification process. 


\subsection{REFERENCES}

Adams, T.L. 1994. Application of the HWVP Measurement Error Model and Feed Test Algorithms to Pilot Scale Feed Testing, PHTD-C93-05.01B, Rev. 0, Pacific Northwest Laboratory, Richland, Washington.

Aitchison, J. 1986. The Statistical Analysis of Compositional Data. Chapman and Hall, New York.

Anderson, D.N., and G.F. Piepel. 1993. Preliminary Investigation of Glass Composition Covariance Matrices and Glass Property Models, PHTD-C92-05.01D, Rev. 0, Pacific Northwest Laboratory, Richland, Washington.

Bryan, M.F. and G.F. Piepel. 1993. Strategy for Product Composition Control in the Hanford Waste Vitrification Plant, PHTD-C93-05.01F, Rev. 0, Pacific Northwest Laboratory, Richland, Washington.

Bryan, M.F. and G.F. Piepel. 1994. Preliminary Feed Test Algorithm for the Hanford Waste Vitrification Plant Product Composition Control System, PHTD-C93-05.01A, Rev. 0, Pacific Northwest Laboratory, Richland, Washington.

Bryan, M.F., G.F. Piepel, and D.B. Simpson. 1994a. Demonstrating Compliance with WAPS 1.3 in the Hanford Waste Vitrification Plant Process, PHTD-C93-05.01K, Pacific Northwest Laboratory, Richland, Washington.

Bryan, M.F., G.F. Piepel, and D.B. Simpson. 1994b. Methods for Estimation of Covariance Matrices and Covariance Components for the Hanford Waste Vitrification Plant Process, PHTD-C93-05.01P, Rev. 0, Pacific Northwest Laboratory, Richland, Washington.

Cochran, W.G. 1977. Sampling Techniques, third edition. John Wiley and Sons, New York.

Edwards, T.B. 1994. "Statistical Analyses of Chemical Composition and Durability Data from DWPF's Startup Test Program (U)," Inter-office Memorandum to J.R. Harbour and M.J. Plodinec, draft dated October 17, 1994, SRT-ASG-940070, Westinghouse Savannah River Company, Aiken, South Carolina.

Hahn, G.J., and W.Q. Meeker. 1991. Statistical Intervals: A Guide for Practitioners. John Wiley and Sons, New York. 
Hrma, P.R., G.F. Piepel, M.J. Schweiger, D.E. Smith, D.-S. Kim, P.E. Redgate, J.D. Vienna, C.A. LoPresti, D.B. Simpson, D.K. Peeler, and M.H. Langowski. 1994.

Property/Composition Relationships for Hanford High-Level Waste Glasses Melting at $\underline{1150^{\circ} \mathrm{C}}$, PVTD-95.02.01B, Pacific Northwest Laboratory, Richland, Washington.

Lindgren, B.W. 1976. Statistical Theorv third edition. MacMillan Publishing Co., Inc., New York.

Postles, R.L. and K.G. Brown. 1991. Savannah River Site Defense Waste Processing Facility Product Composition Control System Statistical Process Control Algorithms, SCS-PMC-91097, Westinghouse Savannah River Company, Aiken, South Carolina..

Randklev, E.R. 1993. Hanford Waste Vitrification Plant Project Waste Form Qualification Program Plan, June 1993, WHC-EP-0522, Westinghouse Hanford Company, Richland, Washington.

Reeve, C.P. 1992a. "PCCS Covariance Matrices Based on DWPF Bench Tests (U)," Inter-office Memorandum to C.T. Randall, June 29, 1992, SCS-ASG-92044, Westinghouse Savannah River Company, Savannah River Technology Center, Aiken, South Carolina.

Reeve, C.P. 1992b. "Statistical Analysis of DWPF Bench Tests: SME Compositions (U)," Inter-office Memorandum to R.S. Beck, November 5, 1992, SCS-ASG-92084, Westinghouse Savannah River Company, Savannah River Technology Center, Aiken, South Carolina.

Reeve, C.P. 1993. "Statistical Analysis of DWPF Prototypic Tests: SME Feed (U)," Inter-office Memorandum to R.S. Beck, June 14, 1993, SCS-ASG-93-0050, Westinghouse Savannah River Company, Savannah River Technology Center, Aiken, South Carolina.

Seàrle, S.R., G. Casella, and C.E. McCulloch. 1992. Variance Components. John Wiley and Sons, Inc., New York.

U.S. Department of Energy. 1993. Waste Acceptance Product Specifications for Vitrified High-Level Waste Forms, EM-WAPS, Rev. 0, February 1993, Office of Environmental Restoration and Waste Management, U.S. Department of Energy, Germantown, Maryland.

WSRC. 1993. Defense Waste Processing Facility Waste Form Oualification Report, Volume 5: Technical Bases for the DWPF Glass Product Control Program, WSRC-IM91-116-5, Westinghouse Savannah River Company, Aiken, South Carolina. 
APPENDIX A

STATISTICAL BACKGROUND 


\section{APPENDIX A}

\section{STATISTICAL BACKGROUND}

For precision and brevity in much of the body of this document, it is necessary to employ some statistical terminology and notation. This appendix introduces the required terminology and notation. Full exposition and explanation of this material is beyond the scope of this appendix, but can be found in most texts on probability and mathematical statistics (e.g., Lindgren 1976). This document also uses the concepts and notation of linear algebra, vectors, and matrices. Some of these concepts and notation are defined below; fuller coverage of this material can be found in books on linear algebra (e.g, Searle 1982).

Statistics is the art and science of making decisions in the face of uncertainty. Accordingly, a major task of statistics is the modelling and characterization of uncertainty. The most common statistical method of modelling uncertainty employs the concept of a random variable. Intuitively, a random variable is a quantity that cannot be measured exactly (either because its value is not fixed or because the measurement process is imperfect). Therefore, the behavior of a random variable is described in terms of the probability that the true value of the random variable exists in some set of possible values. Random variables are often denoted by capital letters, e.g., $\mathrm{X}$, while individual values or realizations of a random variable are often denoted by lower case letters, with a subscript to indicate which observation is being represented. For example, $n$ observations of the random variable $X$ might be denoted $x_{1}, x_{2}, \ldots, x_{n}$, or, equivalently, $x_{i}, i=1, \ldots, n$. A group of $n$ observations may also be represented by a vector, $\underline{x}$.

Two basic types of random variables exist. A discrete random variable is one for which the number of possible values is finite or countably infinite. In many cases, discrete random variables are counts of the number of occurrences of certain events. For example, the number of defective items produced by a manufacturing process can 
range from zero to the number of items produced. A continuous random variable is one for which the number of possible values is uncountably infinite. In many cases, continuous random variables take on values in an interval of possible values. For example, the value of many measured characteristics (length, weight, concentration, viscosity) must lie between some more or less well known lower and upper bounds, but, at least theoretically, the individual measurements may take on any value in the interval. Although many of the concepts discussed below apply to both discrete and continuous random variables, most of the quantities involved in HLW vitrification process/product control are best modelled by continuous random variables; therefore, this presentation focuses on continuous random variables.

\section{A.1 DISTRIBUTION AND DENSITY FUNCTIONS}

Two mathematical functions are useful in describing the behavior of a (continuous) random variable: the distribution (or distribution function), and the density (or density function). To each random variable $\mathrm{X}$, there corresponds a distribution function, $F(x) \equiv \operatorname{Pr}\{X \leq x\}$, ${ }^{(a)}$ the probability that the random variable $X$ is less than or equal to the fixed value $x$. As a function, $F(x)$ is monotonic and nondecreasing. Since for each fixed $x, F(x)$ is a probability, $F(x)$ must lie in the interval $[0,1]$.

The density function, $f(x)$, exists for most of the common statistical distributions. When it exists, the density function is simply the first derivative of the distribution function, i.e., $f(x)=F^{\prime}(x)$. The density function characterizes the local behavior of the random variable. By its nature, $f(x) \geq 0$ for all $x$, and

$$
\int_{-\infty}^{\infty} f(x) d x=1
$$

(a) The symbol "I" should be read as "is defined to be equal to." 
In order to achieve this unit integral, a density function incorporates a normalizing constant (usually a function of the parameters of the distribution, which are discussed below).

Many families of random variables (and the corresponding distributions and densities) have been found useful in statistical applications. For example, the most commonly encountered family of statistical distributions is the family of normal (or Gaussian) distributions. The density function for a normally-distributed random variable $\mathrm{X}$ is

$$
f(x)=\frac{1}{\sigma \sqrt{2} \pi} e^{-(x-\mu)^{2} / 2 \sigma^{2}}
$$

$\left(\mu\right.$ and $\sigma^{2}$ are the parameters of the normal distribution and are discussed further below).

Another important family of random variables is the gamma family. The density function for a random variable $\mathrm{X}$ that follows a gamma distribution is

$$
f(x)=\frac{1}{\Gamma(\alpha) \beta^{\alpha}} x^{\alpha-1} e^{-x / \beta} /(0, \infty)(x) .
$$

where $\alpha$ and $\beta$ are the parameters of the gamma distribution (discussed below) and $\mathrm{I}_{(0, \infty)}(\mathrm{x})$ is zero if $\mathrm{x} \leq 0$ and one otherwise (indicating that a gamma random variable takes on only positive values).

The members of a family of random variables are distinguished by the values of the associated parameters. The parameters of a random variable appear in the density function and are often denoted by lower-case Greek letters. For example, the parameters of the normal density given above are $\mu$ and $\sigma^{2}$, while the parameters of the gamma density given above are $\alpha$ and $\beta$. Often, the dependence of the behavior of a random variable on the associated parameters is shown by a slight modification of notation: for example, the density of a random variable following a normal distribution 
with parameters $\mu$ and $\sigma^{2}$ may be denoted $\mathrm{f}\left(\mathrm{x} \mid \mu, \sigma^{2}\right)$, and the density of a gamma distribution with parameters $\alpha$ and $\beta$ may be denoted $f(x \mid \alpha, \beta)$.

A common statistical shorthand for the phrase "the random variable $\mathrm{X}$ follows a normal distribution with parameters $\mu$ and $\sigma^{2 \prime}$ is "X $\sim N\left(\mu, \sigma^{2}\right)$." The shorthand for "the random variable $\mathrm{X}$ follows a gamma distribution with parameters $\alpha$ and $\beta$ " is "X $\Gamma(\alpha, \beta) . "$

An important special case of the gamma distribution is the chi-square distribution. This distribution has a single parameter, $f$, known as the degrees of freedom. A chisquare distribution with $f$ degrees of freedom $\left[\chi^{2}(f)\right]$ is simply a gamma distribution with parameters $\mathrm{f} / 2$ and 2 , i.e., the $\Gamma(\mathrm{f} / 2,2)$ distribution.

\section{A.2 MEAN AND VARIANCE}

The expectation of a function, $\mathrm{h}(\mathrm{X})$, of the random variable $\mathrm{X}$ is defined as:

$$
E(h(x)) \equiv \int_{-\infty}^{\infty} h(x) d F(x)=\int_{-\infty}^{\infty} h(x) f(x) d x
$$

(the last expression makes sense only if the density function exists). Several such functions are important enough to warrant specific names. The mean of a random variable $\mathrm{X}$ is defined as:

$$
\mu_{x} \equiv E(X) \equiv \int_{-\infty}^{\infty} x d F(x)
$$

The mean of a random variable is a measure of the central value (or central tendency) of the random variable. The most common measures of dispersion about this central value are the variance:

$$
\sigma_{X}^{2} \equiv E\left(X-\mu_{X}\right)^{2} \equiv \int_{-\infty}^{\infty}\left(x-\mu_{X}\right)^{2} d F(x)
$$

and the closely related standard deviation: 


$$
\sigma_{X} \equiv \sqrt{\sigma_{X}^{2}}
$$

(When the meaning is clear from context, the subscripts on $\mu_{X}, \sigma_{X}{ }^{2}$, and $\sigma_{x}$ may be omitted.) Due to the simple relationship between variance and standard deviation, much of the discussion (though not, of course, the equations) in this appendix could be framed in terms of either quantity, and shifts between variance and standard deviation go unremarked henceforth.

The mean and variance are examples of moments of a distribution. Moments are simply expectations of powers of the random variable (often centered by subtracting the mean). The moments of a distribution convey information on the location and shape of the distribution and hence on the behavior of the random variable. The first moment of a distribution is the mean and, as mentioned above, is a measure of the central value (location) of the distribution. The second (central) moment is the variance and hence is a measure of the spread (scale) of the distribution. The third moment measures the skewness of the distribution, and the fourth moment measures kurtosis (how "heavytailed" and peaked the distribution is).

The moments of a distribution are not usually the parameters of the distribution. The exception is the normal distribution, for which the parameters $\mu$ and $\sigma^{2}$ are indeed the mean and variance, respectively. The mean and variance of many distributions are simple functions of the parameters. For example, the mean and variance of a $\Gamma(\alpha, \beta)$ distribution are $\alpha \beta$ and $\alpha \beta^{2}$, respectively; the mean and variance of a chi-square distribution with $f$ degrees of freedom are $f$ and $2 f$, respectively.

In some cases, it is useful to specify only the mean and variance of a random variable, without ascribing to it a distributional form (such as normal or gamma). In this case, an adaptation of the shorthand above is employed -- "X $-\left(\mu, \sigma^{2}\right)^{n}$ means that $\mathrm{X}$ is a random variable with mean $\mu$ and variance $\sigma^{2}$. 


\section{A.3 MULTIVARLATE DATA, COVARIANCE, AND CORRELATION}

The discussion of random variables above concentrated on the univariate situation, i.e., the modelling of a single quantity (even though many measurements or observations of that quantity may be available). However, in many situations, 'he simultaneous behavior of several different quantities is of interest. This is the multivariate situation. The obvious example here is the composition of a vitrification process batch. For use in melt/glass property models, batch composition is usually expressed as mass fractions (proportions or percentages) of nine individual oxides $\left(\mathrm{SiO}_{2}, \mathrm{~B}_{2} \mathrm{O}_{3}, \mathrm{Na}_{2} \mathrm{O}, \mathrm{Li}_{2} \mathrm{O}, \mathrm{CaO}\right.$, $\mathrm{MgO}, \mathrm{Fe}_{2} \mathrm{O}_{3}, \mathrm{Al}_{2} \mathrm{O}_{3}, \mathrm{ZrO}_{2}$ ) and a catchall tenth category, Others. Since these mass fractions must sum to one, they are obviously not independent of one another; hence their simultaneous behavior is of interest.

In multivariate statistics, subscripts are used to distinguish between different random variables. For example, the 10 components of a vitrification process batch can be denoted by $\mathrm{X}_{1}, \mathrm{X}_{2}, \ldots, \mathrm{X}_{10}$. Individual observations of a single random variable are usually indicated by a second subscript; for example, $x_{i j}$ is the $j$-th observation of the $i$-th random variable.

Most of the standard univariate distributions and densities have multivariate generalizations. When modelling several random variables simultaneously, joint distributions and joint densities, which are functions that model the simultaneous probabilistic behavior of the variables, must be considered. In addition, when examining the effects of one variable on another, conditional distributions and conditional densities, which model the probabilistic behavior of one or more variables given the values of other variables, become important. The notation can get quite complex, so, rather than attempting a general treatment, notation is introduced below only as necessary.

In multivariate statistics, the tendency of several quantities to vary together ("covary") is of interest. The statistical covariance between two random variables $X_{i}$ and $X_{j}$ 
is defined as:

$$
\sigma_{i j} \equiv E\left(X_{i}-\mu_{i}\right)\left(X_{j}-\mu_{j}\right)
$$

where the expectation is taken with respect to the joint distribution of $X_{i}$ and $X_{j}$ (i.e., this is a double integral). Whereas the variance of a random variable must be nonnegative (by definition), the covariance between two random variables can be positive, negative, or zero. Positive covariance indicates that the two variables tend to. vary together; i.e., if one is large (relative to its mean), the other tends also to be large, and if one is small, the other tends to be small. (The repetitive use of the word "tend" is necessitated by the probabilistic nature of the behavior of random variables.) Negative covariance indicates that the two variables tend to vary "in opposite directions;" i.e., if one is large (relative to its mean), the other tends to be small (relative to its mean), and vice versa. Zero covariance indicates that the behavior of one variable does not affect the behavior of the other. (a)

Covariances are not scale-invariant, and their magnitudes are affected by the variances of the random variables involved. These characteristics complicate interpretation and comparison of covariances. Statistical correlation is essentially a standardized, unitless covariance. The correlation between $\mathrm{X}_{\mathrm{i}}$ and $\mathrm{X}_{\mathrm{j}}$ is defined as:

$$
\rho_{i j} \equiv \frac{\sigma_{i j}}{\sqrt{\sigma_{i}^{2} \sigma_{j}^{2}}}
$$

Correlations must lie in the interval $[-1,1]$. Interpretation of the sign of a correlation is similar to that for a covariance. In addition, the closer the correlation is to 1 (or -1 ), the

(a) This is not strictly true. Statistical covariance is actually a measure of linear covariance, so a strongly curved relation between two random variables is not necessarily reflected in the standard definition of covariance. It is in fact possible to construct two random variables with zero covariance, even though one is an exact function of the other. 
nearer the relationship between the two variables is to perfect linearity. The correlation between two random variables is zero if and only if the covariance between these two variables is zero. Two variables that have zero correlation (covariance) are said to be uncorrelated; if the correlation (covariance) is non-zero, the two variables are said to be correlated. Correlated observations are not independent.

Matrix notation is quite useful in multivariate statistics. In this document, matrices are denoted by upper case letters (e.g., $\Sigma$ or $S$ ), and symbols for vectors are underlined (e.g., $\underline{\mu}$ ). The random vector, $\underline{X}$, is a vector of random variables, $X_{i}, i=1, \ldots$, p. The associated mean vector (the vector of means of the individual random variables) is denoted by $\mu$. A convenient method for summarizing the variances and pairwise covariances of the elements of the random vector $\underline{X}$ is the variance-covariance matrix (for brevity, called the covariance matrix below):

$$
\Sigma \equiv\left[\begin{array}{ccccc}
\sigma_{1}^{2} & \sigma_{12} & \sigma_{13} & \cdots & \sigma_{1 p} \\
\sigma_{21} & \sigma_{2}^{2} & \sigma_{23} & \cdots & \sigma_{2 p} \\
\sigma_{31} & \sigma_{32} & \sigma_{3}^{2} & \cdots & \sigma_{3 p} \\
\vdots & \vdots & \vdots & \ddots & \vdots \\
\sigma_{p 1} & \sigma_{p 2} & \sigma_{p 3} & \cdots & \sigma_{p}^{2}
\end{array}\right]
$$

The covariance matrix contains the variances of the individual random variables in the diagonal positions and the pairwise covariances in the off-diagonal positions. As a consequence of the definition of covariance, the covariance matrix is symmetric (i.e., $\sigma_{\mathrm{ij}}=\sigma_{\mathrm{ji}}$ ). If the underlying random vector has $\mathrm{p}$ elements, the covariance matrix has $\mathrm{p}$ rows and $\mathrm{p}$ columns; i.e., its dimension is $\mathrm{p} \times \mathrm{p}$. 
Correlations can also be represented in matrix form; the correlation matrix is defined as:

$$
P \equiv\left[\begin{array}{ccccc}
1 & \rho_{12} & \rho_{13} & \cdots & \rho_{1 p} \\
\rho_{21} & 1 & \rho_{23} & \cdots & \rho_{2 p} \\
\rho_{31} & \rho_{32} & 1 & \cdots & \rho_{3 p} \\
\vdots & \vdots & \vdots & \ddots & \vdots \\
\rho_{p 1} & \rho_{p 2} & \rho_{p 3} & \cdots & 1
\end{array}\right] .
$$

The diagonal elements of a correlation matrix are always one (since, by definition, the correlation of a random variable with itself is one); the pairwise correlations appear in the off-diagonal positions. Like the covariance matrix, the correlation matrix is symmetric $\left(\rho_{\mathrm{ij}}=\rho_{\mathrm{ji}}\right)$.

Multivariate generalizations of many common statistical distributions exist. The notation used to specify the (joint) distribution associated with a random vector $\underline{X}$ parallels that used for a univariate random variable. For example, "X $-\operatorname{MVN}(\underline{\mu}, \Sigma)$ " indicates that the random vector $\underline{X}$ follows a multivariate normal distribution with parameters $\underline{\mu}$ and $\Sigma$. " $\underline{X}-(\underline{\mu}, \Sigma)$ " indicates that $\underline{X}$ follows a (multivariate) distribution with mean vector $\mu$ and covariance matrix $\Sigma$.

\section{A.4 ESTIMATING POPULATION PARAMETERS WTTH SAMPLE STATISTICS}

Up to this point, various statistical distributions, parameters, and other theoretical constructs used to model the behavior of random variables have been defined and discussed. In much of statistics, such models for some population (real or abstract) of items are postulated or hypothesized, and information is collected about a sample drawn from this population. The objectives of this activity include checking the models, estimating parameters, and drawing inferences about the population, based on the sample. Estimation often involves calculating sample analogues to population parameters, moments, and other characteristics. Some of these estimation procedures, and the associated notation, are discussed below. 
The usual assumption about a sample is that it is drawn at random from the underiying population. The technical definition of a random sample is somewhat involved, but essentially a random sample is one in which each item in the population has an equal chance of being selected. A related concept is that of independent and identically distributed (IID) observations. Given a sample of size $n, x_{i}, i=1, \ldots, n$, the assumption might be that each $x_{i}$ is a realization of a single random variable $X$, or, equivalently, that the distribution of $X_{i}$ is the same for all $i$. This is the concept of identically distributed observations. The concept of independence is essentially that the value of $X_{i}$ is unaffected by the values of any of the other $X_{j} s(j \neq i)$. The statistical shorthand used to describe this situation is " $X_{i}, i=1, \ldots, n-\operatorname{IID} D(p)$," where $D$ is the assumed distribution and $\mathrm{p}$ is the vector of parameters of $\mathrm{D}$. One link between random sampling and IID observations is this: if $D(p)$ is the statistical distribution for a given population, and $X_{i}, i=1, \ldots, n$, is a random sample from the population, then $X_{i}, i=1$, ..., $\mathrm{n} \sim \mathrm{IID} \mathrm{D}(\mathrm{p})$.

Assume that a random sample of size $\mathrm{n}$ is available from a population with mean $\mu$ and variance $\sigma^{2}$; i.e., $X_{j}, \mathrm{i}=1, \ldots, \mathrm{n}, \sim \operatorname{IID}\left(\mu, \sigma^{2}\right)$. The sample-based estimate of the population mean, $\mu$, is the sample mean:

$$
\bar{x} \equiv \frac{1}{n} \sum_{i=1}^{n} x_{i}
$$

The sample-based estimate of the population variance, $\sigma^{2}$, is the sample variance:

$$
s_{x}^{2} \equiv \frac{1}{n-1} \sum_{i=1}^{n}\left(x_{i}-\bar{x}\right)^{2} .
$$


The sample-based estimate of the population standard deviation, $\sigma$, is the sample standard deviation:

$$
s_{x} \equiv \sqrt{s_{x}^{2}} .
$$

The sample mean, $\bar{X}$, is a point estimator of the population mean, $\mu$. In many situations, both a point estimate of the population mean and some idea of the quality of this estimate are required. To address this issue, it must be recognized that the sample mean is a random variable, since it is a function of the random variables $X_{i}, i=1, \ldots, n$. Therefore, the sample mean has an associated mean and variance. It can be shown that the sample mean is unbiased, i.e., that $\mathrm{E}(\overline{\mathrm{X}})=\mu$, so the question of the quality of the sample mean as an estimator of the population mean comes down to the uncertainty in the sample mean. This uncertainty is measured by the standard deviation (or the variance) of $\bar{X}$. In a wide range of cases, the standard deviation of $\overline{\mathrm{X}}$ is well estimated by

$$
s_{\bar{X}}=\frac{s_{X}}{\sqrt{n}} .
$$

This quantity, also known the standard error ${ }^{(a)}$ of the mean, is used to construct confidence intervals for the population mean.

The preceding discussion of the standard error of the mean is important to inference for the population mean, but it also serves to illustrate that statistical estimators, such as the sample mean, variance, and standard deviation, are random variables and thus have associated uncertainty. This uncertainty must be quantified in order to judge the quality of the estimators and to draw inferences about true

(a) The term "standard error" is often used to refer to the standard deviation of an estimator, as opposed to the standard deviation associated with individual observations. 
(population) values. The HLW vitrification process/product control algorithms must deal with uncertainties in statistical estimators, as well as with uncertainties in data.

In the multivariate case, each observation is a vector (rather than a single number). For example, if interest focuses on $p$ characteristics of each item and $n$ items are examined, the data comprise $n$ vectors, each of length $\mathrm{p}$. Denote the observed value for the $j$-th characteristic of the $i$-th item as $x_{i j}$, where $j=1, \ldots, p$, and $i=1, \ldots, n$, and assume that the observations are IID. The sample-based estimate of the population covariance between characteristics $\mathrm{j}$ and $\mathrm{k}, \sigma_{\mathrm{jk}}$, is the sample covariance:

$$
\hat{\sigma}_{j k} \equiv s_{j k} \equiv \frac{1}{n-1} \sum_{i=1}^{n}\left(x_{i j}-\bar{x}_{j}\right)\left(x_{i k}-\bar{x}_{k}\right)
$$

where $\bar{x}_{j}$ and $\bar{x}_{k}$ are the sample means of the $j$-th and $k$-th characteristics, respectively. The sample-based estimate of the population covariance matrix, $\Sigma$, is the sample covariance matrix:

$$
S \equiv\left[\begin{array}{ccccc}
s_{1}^{2} & s_{12} & s_{13} & \cdots & s_{1 p} \\
s_{21} & s_{2}^{2} & s_{23} & \cdots & s_{2 p} \\
s_{31} & s_{32} & s_{3}^{2} & \cdots & s_{3 p} \\
\vdots & \vdots & \vdots & \ddots & \vdots \\
s_{p 1} & s_{p 2} & s_{p 3} & \cdots & s_{p}^{2}
\end{array}\right]=\frac{1}{n-1} \sum_{i=1}^{n}\left(\underline{x}_{i}-\underline{\underline{x}}\right)\left(\underline{x}_{i}-\underline{\underline{x}}\right)^{\top}
$$

where $\underline{x}_{i}$ is the $i$-th observation (a column vector containing the observed values of the $p$ characteristics for the $i$-th item), $\overline{\underline{x}}$ is the column vector containing the sample means for the $\mathrm{p}$ characteristics, and the superscript " $T$ " indicates vector transpose. Since there are $\mathrm{p}$ characteristics, the sample covariance matrix is a $\mathrm{p} \times \mathrm{p}$ matrix, and, like the population covariance matrix, it is symmetric. The elements of the sample covariance matrix may be computed individually [using the formula for single sample covariances given in 
Equation (A.16)], or the whole matrix may be computed using the vector formula given in Equation (A.17). These methods are equivalent (unless there are missing data).

The sample-based estimate of the population correlation between characteristics $i$ and $j, \rho_{i j}$, is the sample correlation:

$$
\hat{\rho}_{i j} \equiv r_{i j} \equiv \frac{s_{i j}}{\sqrt{s_{i}^{2} s_{j}^{2}}}
$$

The sample-based estimate of the population correlation matrix, $\mathrm{P}$, is the $\mathrm{p} \times \mathrm{p}$ symmetric sample correlation matrix:

$$
R \equiv\left[\begin{array}{ccccc}
1 & r_{12} & r_{13} & \cdots & r_{1 p} \\
r_{21} & 1 & r_{23} & \cdots & r_{2 p} \\
r_{31} & r_{32} & 1 & \cdots & r_{3 p} \\
\vdots & \vdots & \vdots & \ddots & \vdots \\
r_{p 1} & r_{p 2} & r_{p 3} & \cdots & 1
\end{array}\right]
$$

\section{A.5 PROPERTY MODEL NOTATION}

Several property models being developed by CVS are second-order mixture models, the general form of which is

$$
\phi_{k}=\sum_{i=1}^{10} b_{i k} x_{i}+\sum_{i=1}^{9} \sum_{j>i}^{10} b_{i j k} x_{i} x_{j}
$$

where $\phi_{\mathrm{k}}$ is the $\mathrm{k}$-th melt/glass property (or, in some cases, a simple mathematical transformation thereof), $x_{j}$ and $x_{j}$ are the mass fractions of the $i$-th and $j$-th oxides, and $b_{i k}$ and $b_{i j k}$ are the coefficients of the relation between the oxide mass fractions and $\phi_{k}$ 
(to be estimated from the CVS database). The oxide mass fractions used in a mixture model must sum to one, that is,

$$
\sum_{i=1}^{10} x_{i}=1
$$

Some of the models developed by CVS are first-order, meaning that, for some properties $(k), b_{i j k}=0$ for all $i$ and $j$. The form of a first-order model is

$$
\phi_{k}=\sum_{i=1}^{10} b_{i k} x_{i}
$$

Both the first-order model and the second-order model can be written in the form:

$$
\phi_{k}=\underline{x}^{T} \underline{b}_{k}
$$

where $\underline{x}$ is the vector containing the oxide mass fractions (and cross-products thereof, if the model is second-order), and $\underline{b}_{k}$ is the vector of estimated coefficients relating these composition data to the $\mathrm{k}$-th property. Such models are linear in the estimated coefficients, $\underline{b}_{k}$. First-order models are also linear in the data, $\underline{x}$.

\section{A.6 REFERENCES}

Lindgren, B.W. 1976. Statistical Theory, third edition. MacMillan Publishing Co., Inc., New York.

Searle, S.R. 1982. Matrix Algebra Useful for Statistics. John Wiley and Sons, New York. 
APPENDIX B

ESTIMATING AND MANIPULATING UNCERTAINTIES 
(2) 


\section{APPENDLX B}

\section{ESTIMATING AND MANIPULATING UNCERTAINTIES}

Data from the high-level waste ( $\mathrm{HLW}$ ) vitrification process will be multivariate (e.g., feed compositions) and will be subject to several sources of uncertainty. Requirements and specifications imposed on HLW vitrification material apply to univariate attributes, many of which will be calculated from (multivariate) feed compositions using empirical models developed as part of the Composition Variability Study (CVS; Hrma, Piepel, et al. 1994). Therefore, proper inference in HLW vitrification process/product control requires

- estimating univariate and multivariate uncertainties and components thereof (e.g., variances, variance components, covariance matrices, covariance components);

- propagating multivariate uncertainties to yield univariate uncertainties;

- combining univariate uncertainties to yield an estimate of overall uncertainty in an estimated attribute value, and assigning a measure of strength of belief to this overall uncertainty estimate; and

- updating existing uncertainty estimates to reflect both prior and current information.

These topics are discussed in the following sections. This appendix supplies the technical details that do not appear in Section 4. Expanded treatments of these subjects appear in Bryan and Piepel (1994), Bryan, Piepel, and Simpson (1994), and Searle, Casella, and McCulloch (1992). Basic statistical concepts and notation are covered in Appendix A. 


\section{B.1 THE MODEL FOR COMPOSITION UNCERTAINTY IN HLW VITRIFICATION DATA}

Let $Y_{\mathrm{ijk}}$ represent the value of some attribute estimated from the $\mathrm{k}$-th analysis of the $\mathrm{j}$-th sample from the $\mathrm{i}$-th batch of HLW vitrification process material. ${ }^{(a)} \mathrm{Y}_{\mathrm{ijk}}$ is subject to model uncertainty and to three sources of composition uncertainty (batch-tobatch variability, within-batch uncertainty, and analytical uncertainty). Model uncertainty will be estimated separately and then combined with composition uncertainty (see Sections B.5 and B.6). Composition uncertainty (and its components) will be estimated using the following model:

$$
Y_{i j k}=\mu+\beta_{i}+\omega_{i j}+\alpha_{i j k},
$$

where $\beta_{\mathrm{i}}-\left(0, \sigma_{\beta}{ }^{2}\right), \omega_{\mathrm{ij}}-\left(0, \sigma_{\omega}{ }^{2}\right), \alpha_{\mathrm{ijk}} \sim\left(0, \sigma_{\alpha}{ }^{2}\right)$, and all the random variables are uncorrelated. In this model, $\sigma_{\beta}{ }^{2}$ represents batch-to-batch variability, $\sigma_{\omega}{ }^{2}$ represents within-batch uncertainty, and $\sigma_{\alpha}{ }^{2}$ represents analytical uncertainty. The $\beta_{\mathrm{i}}$, $\omega_{\mathrm{ij}}$, and $\alpha_{\mathrm{ijk}}$ are known as random effects. The quantities $\sigma_{\beta}{ }^{2}, \sigma_{\omega}{ }^{2}$, and $\sigma_{\alpha}{ }^{2}$ (and estimates of these quantities) are known as variance components (or components of variance).

The number of batches in a single waste type is denoted by $b$, the number of samples taken from the $\mathrm{i}$-th batch is denoted by $\mathrm{w}_{\mathrm{i}}$, ${ }^{(b)}$ and the number of analyses performed on the $\mathrm{j}$-th sample from the $\mathrm{i}$-th batch is denoted by $\mathrm{a}_{\mathrm{ij}}$. If $\mathrm{w}_{\mathrm{i}}=\mathrm{w}$ for all $\mathrm{i}$, and $\mathrm{a}_{\mathrm{ij}}=\mathrm{a}$ for all $\mathrm{i}$ and $\mathrm{j}$, the data are said to be balanced. Otherwise, the data are said to be unbalanced.

(a) Waste to be processed by the. HLW vitrification plant will be classified into waste types. However, all requirements apply within a single waste type; no inferences are required across waste types. For this reason, the model and methods discussed here should be applied separately to each waste type. Therefore, the notation is simplified by not accounting for waste type.

(b) It may seem more intuitive to use $s_{i}$ for the number of samples taken from the ith batch. However, the letter $s$ (and subscripted versions thereof) is reserved in this document for denoting a standard deviation. Therefore, $w_{i}$ is used to denote the number of samples within a batch. 
Several of the equations and methods discussed below rely on the within-sample mean, $\overline{\mathrm{Y}}_{\mathrm{ij}}$. The within-sample mean is the mean over the $\mathrm{a}_{\mathrm{ij}}$ analyses performed on the $j$-th sample from the $i$-th batch:

$$
\bar{Y}_{i j \bullet} \equiv \frac{1}{a_{i j}} \sum_{k=1}^{a_{i j}} Y_{i j k} .
$$

\section{B.2 THE BATCH MEAN AND ASSOCIATED UNCERTAINTIES}

Under the model given in Equation (B.1), the true attribute value for the $i$-th batch is $\mu+\beta_{\mathrm{i}}$. To check a single-batch requirement on this attribute, $\mu+\beta_{\mathrm{i}}$ will be estimated with the batch mean. ${ }^{(a)}$ In general, the $\mathrm{i}$-th batch mean is

$$
\bar{Y}_{i \infty} \equiv \frac{1}{w_{i j}} \sum_{i=1}^{w_{i}} \bar{Y}_{i j \cdot}=\frac{1}{w_{i j}} \sum_{j=1}^{w_{i}}\left[\frac{1}{a_{i j}} \sum_{k=1}^{a_{i j}} Y_{i j k}\right] \text {; }
$$

that is, the batch mean is a mean of $w_{i}$ within-sample means from the $\mathrm{i}$-th batch. By substituting for $\mathrm{Y}_{\mathrm{ijk}}$ from Equation (B.1) and simplifying, it can be shown that

$$
\bar{Y}_{i \bullet \bullet}=\mu+\beta_{i}+\frac{1}{w_{i j}} \sum_{i=1}^{w_{i}} \omega_{i j}+\frac{1}{w_{i j}} \sum_{i=1}^{w_{i}}\left[\frac{1}{a_{i j}} \sum_{k=1}^{a_{i j}} \alpha_{i j k}\right] .
$$

(a) Since the batch effect is assumed to be random rather than fixed, an argument can be made that the best estimator for the quantity $\mu+\beta_{i}$ is not the batch mean value, but a "shrunken" version of this value. Searle et al. (1992, Chapter 7, esp. pp. 258-260) discuss this problem. The preliminary Feed Test Algorithm (Bryan and Piepel 1994) ignores this complication. If testing of the HLW vitrification process/product control algorithms with the Plant Simulation Code indicates problems, this issue should be re-examined. 
For balanced data, these expressions simplify as follows:

$$
\bar{Y}_{i \bullet} \equiv \frac{1}{w a} \sum_{j=1}^{w} \sum_{k=1}^{a} Y_{i j k}=\mu+\beta_{i}+\frac{1}{w} \sum_{j=1}^{w} \omega_{i j}+\frac{1}{w a} \sum_{j=1}^{w} \sum_{k=1}^{a} \alpha_{i j k} .
$$

Since the target of inference for a single-batch requirement is $\mu+\beta_{\mathrm{i}}$ (the true attribute value in the i-th batch), inference should be conditional on (i.e., taking as fixed) the true value of $\beta_{\mathrm{i}}$. Thus, for testing a single-batch requirement, the estimate of composition uncertainty in the batch mean must account for uncertainties due to $\omega_{\mathrm{ij}}$ and $\alpha_{\mathrm{ijk}}$ (the within-batch and analytical uncertainties, respectively), but uncertainty in $\beta_{\mathrm{i}}$ (the batch-to-batch uncertainty) is irrelevant.

Since the batch mean is a mean of within-sample means, each within-sample mean can be viewed as an observation contributing to the batch mean. In other words, the population underlying the batch mean is the population of within-sample means, $\bar{Y}_{\mathrm{ij}}$ • Therefore, the uncertainty in the within-sample means is important in making inferences about the batch mean. In general, the (conditional) composition uncertainty associated with the $\mathrm{j}$-th within-sample mean from the $\mathrm{i}$-th batch is

$$
\operatorname{Var}\left(\bar{Y}_{i j} \mid \beta_{i}\right)=\sigma_{\omega}^{2}+\frac{\sigma_{\alpha}^{2}}{a_{i j}}
$$

For balanced data, this composition uncertainty is

$$
\operatorname{Var}\left(\bar{Y}_{i j} \mid \beta_{i}\right)=\sigma_{\omega}^{2}+\frac{\sigma_{\alpha}^{2}}{a}
$$

In general, the (conditional) composition uncertainty in the $\mathrm{i}$-th batch mean is

$$
\operatorname{Var}\left(\bar{Y}_{i \bullet} \mid \beta_{i}\right)=\frac{\sigma_{\omega}^{2}}{w_{i}}+\frac{\sigma_{\alpha}^{2}}{w_{i} \bar{a}_{i}},
$$


where

$$
\bar{a}_{i} \equiv\left(\frac{1}{w_{i j}} \sum_{i}^{w_{i}} \frac{1}{a_{i j}}\right)^{-1} .
$$

For balanced data, the (conditional) composition uncertainty in the i-th batch mean is

$$
\operatorname{Var}\left(\bar{Y}_{i \infty} \mid \beta_{i}\right)=\frac{\sigma_{\omega}^{2}}{w}+\frac{\sigma_{\alpha}^{2}}{w a}
$$

Estimating these uncertainties is discussed in Section B.4.

Recognizing the batch mean as a mean of within-sample means assists in identifying the proper sample size to be associated with the batch mean when constructing confidence and/or tolerance limits. For example, in the balanced case, the number of individual $\mathrm{Y}_{\mathrm{ijk}}$ involved in calculating the batch mean is wa, but this is not the proper sample size for statistical inference. The proper sample size is equal to the number of within-sample means involved in constructing the batch mean. This sample size is $w$, in the balanced case, or $w_{i}$, in the unbalanced case.

Model uncertainty must also be accounted for by the statistical inference procedure. Estimating model uncertainty is discussed in Section B.5. Combining model and composition uncertainties is discussed in Section B.6.

\section{B.3 THE MULTIPLE-BATCH MEAN AND ASSOCLATED UNCERTAINTIES}

The target of inference for a multiple-batch requirement is a percentile of the distribution of batch means. Under the model given in Equation (B.1) and the assumptions pertaining thereto, the true batch means, $\mu+\beta_{\mathrm{i}}$, follow a distribution with mean $\mu$ and variance $\sigma_{\beta}{ }^{2}$. Therefore, estimates of $\mu$ and $\sigma_{\beta}{ }^{2}$ are required for statistical inference. 
The procedure for checking multiple-batch requirements will use the multiplebatch mean, $\bar{Y}_{\ldots . . .}$ as an estimate of $\mu$. The multiple-batch mean is defined as

$$
\bar{Y}_{\infty \infty} \equiv \frac{1}{b} \sum_{i=1}^{b} \bar{Y}_{i \infty}=\frac{1}{b} \sum_{i=1}^{b}\left[\frac{1}{w_{i j}} \sum_{i=1}^{w_{i}} \bar{Y}_{i j \cdot}\right] .
$$

The multiple-batch mean is a mean of the $b$ individual batch means. By substituting for $\mathrm{Y}_{\mathrm{ijk}}$ from Equation (B.1) and simplifying, it can be shown that

$$
\bar{Y}_{\text {oo }}=\mu+\frac{1}{b} \sum_{i=1}^{b} \beta_{i}+\frac{1}{b} \sum_{i=1}^{b}\left[\frac{1}{w_{i j}} \sum_{i}^{w_{i}}\left(\omega_{i j}+\frac{1}{a_{i j}} \sum_{k=1}^{a_{i j}} \alpha_{i j k}\right)\right] .
$$

For balanced data, these expressions simplify as follows:

$$
\begin{gathered}
\bar{Y}_{\ldots}=\frac{1}{b w a} \sum_{i=1}^{b} \sum_{j=1}^{w} \sum_{k=1}^{a} Y_{i j k} \\
=\mu+\frac{1}{b} \sum_{i=1}^{b} \beta_{i}+\frac{1}{b w} \sum_{i=1}^{b} \sum_{j=1}^{w} \omega_{i j}+\frac{1}{b w a} \sum_{i=1}^{b} \sum_{j=1}^{w} \sum_{k=1}^{a} \alpha_{i j k} .
\end{gathered}
$$

As stated above, the statistical procedure for checking multiple-batch requirements requires an estimate of $\sigma_{\beta}{ }^{2}$. Estimating $\sigma_{\beta}{ }^{2}$ is discussed in Section B.4. It is instructive to consider the uncertainty in the empirical batch means. In general, the composition uncertainty in the $\mathrm{i}$-th batch mean is

$$
\operatorname{Var}\left(\bar{Y}_{i \infty}\right)=\sigma_{\beta}^{2}+\frac{\sigma_{\omega}^{2}}{w_{i}}+\frac{\sigma_{\alpha}^{2}}{w_{i} \bar{a}_{i}},
$$


where

$$
\bar{a}_{i} \equiv\left(\frac{1}{w_{i}} \sum_{j=1}^{w_{i}} \frac{1}{a_{i j}}\right)^{-1} .
$$

For balanced data, the composition uncertainty in the i-th batch mean is

$$
\operatorname{Var}\left(\bar{Y}_{i \infty}\right)=\sigma_{\beta}^{2}+\frac{\sigma_{\omega}^{2}}{w}+\frac{\sigma_{\alpha}^{2}}{w a} .
$$

Thus, the empirical batch means, $\mathrm{Y}_{\mathrm{i} m}$, have a larger variance than do the true batch means, $\mu+\beta_{i}$. This variance can also be estimated using the methods discussed in Section B.4.

The proper estimate of composition variability to be used in the inferential procedure may require more investigation. This issue is related to the issue of removal of nuisance uncertainties, which is discussed in Section 5. The WAPS 1.3 compliance strategy to be used by the HLW vitrification process/product control system will employ an estimate of the variance that appears in Equation (B.16). This uncertainty is larger than $\sigma_{\beta}{ }^{2}$ alone; hence, inference based on this uncertainty is conservative relative to inference based on an estimate of $\sigma_{\beta}{ }^{2}$. If testing of the WAPS 1.3 compliance strategy (e.g., using the Plant Simulation Code, as discussed by Bryan and Piepel 1993) indicates that this conservatism is likely to hinder verification of WAPS 1.3 compliance, this issue should be revisited.

Recognizing the multiple-batch mean as a mean of individual batch means assists in identifying the proper sample size to be associated with the multiple-batch mean when constructing confidence and/or tolerance limits. For example, in the balanced case, the number of individual $\mathrm{Y}_{\mathrm{ijk}}$ involved in calculating the multiple-batch mean is bwa, but this 
is not the proper sample size for statistical inference. The proper sample size is equal to the number of batch means involved in constructing the multiple-batch mean, $b$.

Model uncertainty must also be accounted for by the statistical inference procedure. Estimating model uncertainty is discussed in Section B.S. Combining model and composition uncertainties is discussed in Section B.6.

\section{B.4 ESTIMATING VARIANCE COMPONENTS}

The model given in Equation (B.1) is an example of a two-way nested random model. The term "nested" is applied because the random effects are hierarchical; for example, uncertainty exists among analyses within a single sample, among samples within a single batch, and among batches within a waste type. Each observed, measured, or estimated attribute value includes uncertainty introduced at each level of this hierarchy. The uncertainty at each level in this hierarchy can be represented by a variance. These hierarchical representations of uncertainty are the variance components.

Methods for estimating variance components are discussed in great detail by Searle et al. (1992). The discussion below focuses on general principles, applicability to HLW vitrification process/product control, and special features of the HLW vitrification process.

Assume that the data available for estimating variance components are balanced. As in Section B.1, the number of batches is denoted by b, the number of samples taken from each batch is denoted by $w$, and the number of analyses run on each sample is denoted by $a$. The total number of observations is then bwa. These data may be analyzed with the analysis of variance (ANOVA) ${ }^{(a)}$, as in Table B.1. The estimates of

(a) The analysis of variance, or ANOVA, is a well-known and widely-used statistical procedure. ANOVA is discussed in most books on basic applied statistics (e.g., Snedecor and Cochran 1980); Graybill (1976) and Searle (1971) present extensive theoretical treatments of ANOVA. 
TABLE B.1. Analysis of Variance Table for the Two-Way Nested Random Model, $\mathrm{Y}_{\mathrm{ijk}}=\mu+\beta_{\mathrm{i}}+\omega_{\mathrm{ij}}+\alpha_{\mathrm{ijk}}$

\begin{tabular}{|c|c|c|c|c|}
\hline $\begin{array}{l}\text { Source of } \\
\text { Variation } \\
\end{array}$ & $\begin{array}{c}\text { Degrees of } \\
\text { Freedom } \\
\end{array}$ & Sum of Squares & Mean Square & $\begin{array}{c}\text { Expected } \\
\text { Mean Square }\end{array}$ \\
\hline Batch-to-Batch & b-1 & $S S B \equiv w a \sum_{i=1}^{b}\left(\bar{y}_{i} \cdot \bar{y}_{\ldots} \ldots\right)^{2}$ & $\mathrm{MSB} \equiv \mathrm{SSB} /(\mathrm{b}-1)$ & $\mathrm{wa} \sigma_{\beta}^{2}+\mathrm{a} \sigma_{\omega}^{2}+\sigma_{\alpha}^{2}$ \\
\hline Within-Batch & $\mathrm{b}(w-1)$ & $S S W \equiv a \sum_{i=1}^{b} \sum_{j=1}^{w}\left(\bar{y}_{i j \cdot}-\bar{y}_{i \bullet \cdot}\right)^{2}$ & $\mathrm{MSW} \equiv \mathrm{SSW} / \mathrm{b}(\mathrm{w}-1)$ & $\mathrm{a} \sigma_{\omega}^{2}+\sigma_{\alpha}^{2}$ \\
\hline Analytical & $\mathrm{bw}(\mathrm{a}-1)$ & $S S A \equiv \sum_{i=1}^{b} \sum_{j=1}^{w} \sum_{k=1}^{a}\left(y_{i j k}-\bar{y}_{i j \cdot}\right)^{2}$ & $\mathrm{MSA} \equiv \mathrm{SSA} / \mathrm{bw}(\mathrm{a}-1)$ & $\sigma_{\alpha}^{2}$ \\
\hline Total & bwa-1 & $S S T \equiv \sum_{i=1}^{b} \sum_{j=1}^{w} \sum_{k=1}^{a}\left(y_{i j k}-\bar{y}_{\ldots . .}\right)^{2}$ & & e \\
\hline
\end{tabular}


the individual variance components are

$$
\hat{\sigma}_{\beta}^{2}=\frac{M S B-M S W}{w a}, \quad \hat{\sigma}_{\omega}^{2}=\frac{M S W-M S A}{a}, \quad \hat{\sigma}_{\alpha}^{2}=M S A,
$$

where the symbols used above are defined in Table 1. These ANOVA-based estimators are derived by setting the sample-based mean squares MSA, MSW, and MSB equal to their expectations (the "Expected Mean Squares" of Table 1) and solving for $\sigma_{\alpha}{ }^{2}, \sigma_{\omega}{ }^{2}$, and $\sigma_{\beta}{ }^{2}$.a) The degrees of freedom associated with the estimates of $\sigma_{\omega}{ }^{2}$ and $\sigma_{\beta}{ }^{2}$ can be calculated from the Satterthwaite approximation (Section B.6).

Once these estimates of variance components have been calculated, they can be used to estimate composition uncertainties (and the associated degrees of freedom) for within-sample means and batch means [Equations (B.6), (B.7), (B.14), and (B.16)]. Note that the required variances are linear functions of the variance components, and that the variance component estimators given in Equation (B.17) are linear functions of the mean squares. Therefore, for balanced data, the required variances can be estimated with linear functions of the mean squares (skipping the intermediate estimation of variance components). Such a simplification of the estimation process should be used when available, since a single-step Satterthwaite approximation to the associated degrees of freedom will be more accurate than a two-step approximation. In fact, for balanced data, the estimated variances collapse to simple functions of the mean squares in Table 1 :

$$
\hat{V}\left(\bar{Y}_{i j} \mid \beta_{i}\right)=\frac{M S W}{a}, \quad \hat{V}\left(\bar{Y}_{i \infty}\right)=\frac{M S B}{w a} .
$$

(a) The method of moments is a technique for deriving statistical estimators in which sample-based quantities are set equal to their expectations and the resulting equations are solved for the parameters in terms of the sample-based quantities (Lindgren 1976). Thus, the ANOVA-based estimators discussed here are examples of method of moments estimators. 
The degrees of freedom associated with these estimated variances are simply those associated with the corresponding mean squares, $b(w-1)$ and $b-1$, respectively.

The estimators of variance components given in Equation (B.17) belong to the

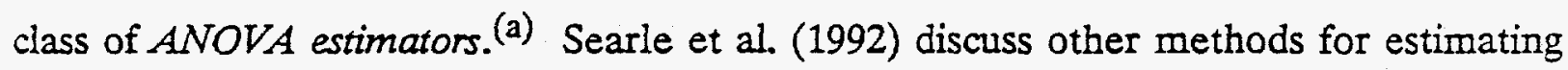
variance components, including maximum likelihood estimation, restricted maximum likelihood estimation, and Bayes procedures. Complete enumeration and elucidation of the wide variety of techniques for variance component estimation are beyond the scope of this document. The choice of estimation technique must depend on the structure of the available data and the assumptions about the process that are considered realistic at the time. Therefore, this choice should be made when data become available, as part of the data analysis process. Some considerations in the choice of specific technique are discussed below.

It is common to assume that random effects in a linear model follow a normal distribution. In fact, some distributional assumption is required for several of the variance component estimation techniques mentioned above (e.g., maximum likelihood and Bayes procedures). The ANOVA estimators are exceptions -- they are derived simply by equating mean squares to their expected values and therefore depend only on the moments of the underlying distribution(s).

The assumption of balanced data greatly simplifies the form of the ANOVA estimators. In fact, with balanced data, several methods for variance component estimation yield estimates identical or closely related to the ANOVA estimates. Unfortunately, this is not the case if the data are unbalanced; in fact, for unbalanced data, several reasonable types of ANOVA estimators exist. Other estimation techniques

(a) This document follows the convention of Searle et al. (1992) in applying the term "ANOVA estimators" to any estimators derived by applying the method of moments to quantities involved in an ANOVA. In some cases (e.g., when the data are not balanced), different types of ANOVA may be legitimately applied to the same data. Different method of moments may result from these different ANOVAs. Therefore, ANOVA estimators are not necessarily unique.

B.11 
yield unique estimators, but these techniques are iterative in nature and can impose a significant computational burden. If imbalance in the available data is not too large, the ANOVA estimators of Equation (B.17) (possibly modified to account for the imbalance) should provide reasonable approximations to the variance components.

ANOVA Estimates of variance components can be negative. This is troubling, since the true values of variance components, by their nature as variances, must be nonnegative. Searle et al. (1992, pp. 129-131) discuss various options for dealing with this problem. One is simply to set a negative estimate to zero (thereby concluding that the related random effect contributes no uncertainty to the observed value); a second is to use one of the methods that guarantee nonnegative estimates (e.g., maximum likelihood and Bayes procedures).

Estimating multivariate composition uncertainties may be required as part of HLW vitrification process/product control. Like univariate uncertainties, multivariate uncertainties may derive from various sources, so methods for estimating components of multivariate uncertainties may be required. Just as for univariate variance components, the importance of multivariate components of uncertainty lies in their crucial role in estimating uncertainty in values (e.g., means) calculated from a set of observations, $\underline{X}_{\mathrm{ijk}}$.

The model given in Equation (B.1) can be generalized to apply to the multivariate situation, e.g., to vectors of measured feed composition:

$$
\underline{X}_{i j k}=\underline{\mu}+\underline{\beta}_{i}+\underline{\omega}_{i j}+\underline{\alpha}_{i j k},
$$

where $\underline{X}_{\mathrm{ijk}}$ is the vector of individual oxide mass fractions $\left(X_{\mathrm{ijk}}, 1=1, \ldots, 10\right)$, and $\underline{\beta}_{\mathrm{i}}, \underline{\omega}_{\mathrm{ij}}$, and $\alpha_{\mathrm{ijk}}$ are vectors of random effects. In this multivariate generalization of Equation (B.1), it is assumed that $\underline{\underline{\beta}}_{\mathrm{i}} \sim\left(\underline{0}, \Sigma_{\beta}\right), \underline{\omega}_{\mathrm{ij}} \sim\left(\underline{0}, \Sigma_{\omega}\right), \underline{\alpha}_{\mathrm{ijk}} \sim\left(\underline{0}, \Sigma_{\alpha}\right)$, and the random vectors $\underline{\beta}_{\mathrm{i}}, \underline{\omega}_{\mathrm{ij}}$ and $\underline{\alpha}_{\mathrm{ijk}}$ are uncorrelated. In analogy to the univariate case, the covariance matrices $\Sigma_{\beta}, \Sigma_{\omega}$, and $\Sigma_{\alpha}$ are known as covariance components (or components of covariance). 
Searle et al. (1992) discuss estimation of covariance components. The method to be used in HLW vitrification process/product control is based on the methods for univariate variance component estimation discussed above and on the well-known formula for the variance of a sum of two random variables (see, for example, Lindgren 1976, p. 137):

$$
\operatorname{Var}\left(X_{i}+X_{j}\right)=\operatorname{Var}\left(X_{i}\right)+\operatorname{Var}\left(X_{j}\right)+2 \operatorname{Cov}\left(X_{i}, X_{j}\right)
$$

from which is easily derived:

$$
\operatorname{Cov}\left(X_{i}, X_{j}\right)=\frac{1}{2}\left\{\operatorname{Var}\left(X_{i}+X_{j}\right)-\left[\operatorname{Var}\left(X_{i}\right)+\operatorname{Var}\left(X_{j}\right)\right]\right\}
$$

To obtain estimates of the components of covariance between $X_{i}$ and $X_{j}$, the three univariate variance components $\left(\sigma_{\beta}{ }^{2}, \sigma_{\omega}{ }^{2}, \sigma_{\alpha}{ }^{2}\right)$ will be estimated for each of $\mathrm{X}_{\mathrm{i}}, \mathrm{X}_{\mathrm{j}}$, and the sum, $X_{i}+X_{j}$, and the above formula will be applied. Performing this estimation for each pair $(i, j), j>i$, "fills in" the upper half of each of the matrices of covariance components; the lower half of each matrix is derived from the symmetricity of covariance matrices.

\section{B.5 PROPAGATING UNCERTAINTIES}

Many of the batch and glass attributes that must be estimated and checked as part of HLW vitrification process/product control will be calculated as functions of more than one uncertain quantity (e.g., oxide mass fractions, other process measurements, empirical model coefficients). In order to check compliance of these attributes with process and product specifications, the total (univariate) uncertainty associated with each attribute must be estimated. Therefore, a procedure for combining multivariate uncertainties (e.g., covariance matrices, covariance components) to yield univariate uncertainties is required.

For HLW process/product control, the multivariate uncertain quantities fall into two categories: 1) composition and other process measurements, and 2) empirical model 
coefficients. As discussed in Section 4.1, composition uncertainty and components thereof will be estimated by using the CVS models to convert individual measurements of feed composition to property values (e.g., PCT results), and then performing univariate variance component estimation for these results (using the methods discussed in Section B.4). However, future developments and investigations may indicate that efficiency could be increased by estimating (multivariate) covariance components for composition and propagating these covariance components into property (PCT) units. If this is the case, the methods discussed in this section can be used to estimate the contribution of composition uncertainty (and the components thereof) to uncertainty in property values. In any case, the methods discussed here will be used to estimate the contribution of model uncertainty to uncertainty in property values.

The procedure described below is one form of error propagation (or propagation of error). This procedure can be used to estimate uncertainty for a wide variety of functions of multivariate uncertain quantities.

The basis for the error propagation method to be used in HLW vitrification process/product control is as follows. Let y represent the characteristic of interest, and assume that $y=f(\underline{z})$, where $\underline{z}$ is a random vector with mean $\underline{\mu}_{z}$ and covariance matrix $\Sigma_{z}$. Then, using a Taylor series expansion about $\mu_{\mathrm{z}}$ to approximate $\mathrm{f}(\underline{z})$, an approximation to the variance of $\mathrm{y}, \sigma_{\mathrm{y}}{ }^{2}$, can be derived:

$$
\sigma_{y}^{2} \approx \underline{d}_{z}^{T} \Sigma_{z} \underline{d}_{z}
$$

where $\underline{d}_{z}$ is the gradient of $f$ (i.e., the vector of partial derivatives with respect to $z$ ), evaluated at the observed value of $\underline{z}$.

The uncertainty associated with a modelled batch or glass property $(\mathrm{y})$ derives from two distinct sources: 1) uncertainty associated with the estimated coefficients ( $\underline{b})$ of 
the empirical model, and 2) uncertainty associated with the estimated composition ( $\underline{x}){ }^{\text {(a) }}$ Model uncertainty will be represented by the covariance matrix, $\Sigma_{b}$, for the vector of estimated model coefficients (which will be obtained from CVS, Hrma, Piepel, et al. 1994). For simplicity of presentation, it is assumed here that a single covariance matrix representing composition uncertainty, $\Sigma_{x}$ is available. The case of several covariance components for feed composition is discussed at the end of this section.

The general method of error propagation discussed above can be applied to the case in which the random vector $\underline{z}$ consists of two distinct subvectors, e.g., the case in which $y=f(\underline{x}, \underline{b})$. Denote the gradients of $f(\underline{x}, \underline{b})$ with respect to $\underline{x}$ and $\underline{b}$ by $\underline{d}_{x}$ and $\underline{d}_{b}$, respectively. If $\underline{x}$ and $\underline{b}$ are uncorrelated random vectors (a reasonable assumption unless $\underline{x}$ is part of the data used to estimate $\underline{b}$ ), the approximate variance of $y$ divides neatly into two parts, one attributable to composition uncertainty, the other attributable to model uncertainty:

$$
\sigma_{y}^{2} \approx \underline{d}_{x}^{T} \Sigma_{x} \underline{d}_{x}+\underline{d}_{b}^{T} \Sigma_{b} \underline{d}_{b}
$$

For the special case where the function $f(\underline{x}, \underline{b})$ is linear in both the data, $\underline{x}$, and the estimated coefficients, $\underline{b}$, this formula takes on an even simpler form. For this case, $y=$ $\underline{x}^{\mathrm{T}} \underline{\mathrm{b}}, \underline{\mathrm{d}}_{\mathrm{x}}=\underline{\mathrm{b}}, \underline{\mathrm{d}}_{\mathrm{b}}=\underline{\mathrm{x}}$, and

$$
\sigma_{y}^{2} \approx \underline{b}^{T} \Sigma_{x} \underline{b}+\underline{x}^{T} \Sigma_{b} \underline{x}
$$

The HLW vitrification process/product control system will estimate the two types of uncertainty (composition uncertainty and model uncertainty) separately. The method of Section B.6 will be used to combine these two types of uncertainty and to assign degrees of freedom to the resulting estimate of overall uncertainty.

(a) If the property model is second-order, the vector $\underline{x}$ contains not only the individual mass fractions, but also some cross-products. 
As currently envisioned, HLW vitrification process/product control will use error propagation only to estimate model uncertainty. However, if the decision is made later to estimate and propagate (multivariate) covariance components for composition, the error propagation methods discussed in this section can be used to estimate the contribution of composition uncertainty (and the components thereof) to uncertainty in property values. The composition covariance components can be propagated separately. Using the method of Section B.6, the resulting univariate variance components can be combined with the estimated model uncertainty to form a univariate estimate of overall uncertainty in property units and to assign an associated number of degrees of freedom.

\section{B.6 COMBINING UNCERTAINTIES}

When observed data are subject to more than one source of uncertainty, proper estimation of the uncertainty in a function (e.g., the mean) of these observations requires combining variance components. In addition, estimating variance components often requires combining mean squares, and the HLW process/product control algorithms must combine model uncertainty with composition uncertainty. In many cases (including all so far identified for HLW vitrification process/product control), the required combination of mean squares or variance components is a weighted sum, where the weights are related to the distribution of sampling effort (e.g., the number of samples per batch and the number of analyses per sample) or strength of belief in the individual variance components. In general, such weighted sums take the form:

$$
s_{c}^{2}=\sum_{j=1}^{p} c_{j} s_{j}^{2}
$$

where $s_{c}{ }^{2}$ is the required combination of the individual variance components, $s_{j}{ }^{2}$, with weights $c_{j}$.

Some measure of the quality of $s_{c}^{2}$ must be available in order to use this estimate to draw inferences. The quality of a variance estimate is often quantified by the 
associated degrees of freedom. The weighted sum above incorporates several variance estimates, each with an associate number of degrees of freedom, $f_{j}$. What number of degrees of freedom should be associated with the combined variance estimate, $s_{c}{ }^{2}$ ? HLW process/product control will use the answer given by Satterthwaite (1946). The degrees of freedom to be associated with $\mathrm{s}_{\mathrm{c}}{ }^{2}$ is

$$
f_{c}=\frac{\left(\sum_{j=1}^{p} c_{j} s_{j}^{2}\right)^{2}}{\sum_{j=1}^{p} \frac{\left(c_{j}{ }_{j}^{2}\right)^{2}}{f_{j}}}=\frac{\left(s_{c}^{2}\right)^{2}}{\sum_{j=1}^{p} \frac{\left(c_{j} s_{j}^{2}\right)^{2}}{f_{j}}} .
$$

Satterthwaite's approximation, as the above formula is known, was derived under the assumption of normality. Caution should be exercised in applying this formula when some of the $c_{j}$ are negative (which is often the case when estimating variance components). Methods and additional requirements in this case are discussed by Gaylor and Hopper (1969), who show that the approximation is adequate when the component (or the sum of the several components) being subtracted is relatively small.

\section{B.7 UPDATING UNCERTAINTIES}

In some cases, an uncertainty estimate may be available from a source external to the actual Hanford HLW vitrification process data. For example, an uncertainty estimate may be available from operations at other sites (e.g., DWPF, WVDP) or from simulations of the HLW vitrification process. It may be desirable to combine this external uncertainty estimate with data from the Hanford HLW vitrification process. Combining an external uncertainty estimate with current data is here referred to as updating the uncertainty estimate. 
If the external uncertainty estimate can be expressed as a variance, $s^{2}$, with an associated number of degrees of freedom, f, the method described in Section B.6 can be used to combine this information with variance estimates derived using the methods of Sections B.4 and B.5. However, if the external uncertainty estimate appears in some other form, another method of combining this estimate with Hanford HLW vitrification process data must be employed. For example, the external uncertainty estimate may appear as an estimated variance, $s^{2}$, with an associated standard deviation, e. ${ }^{(a)}$ This section describes two methods for combining such an external uncertainty estimate with HLW vitrification process data.

The first method is an adaptation of the method in Section B.6. This method relies on the properties of the chi-square distribution to calculate a number of degrees of freedom associated with $\mathrm{s}^{2}$. If $\mathrm{s}^{2}$ is estimated from a random sample of size $\mathrm{n}$ from a normal distribution, the associated random variable $S^{2}$ follows (a multiple of) a chisquare distribution with $f=n-1$ degrees of freedom (Lindgren 1976, p. 334, Theorem 4). Since the mean and variance of a chi-square distribution are $f$ and $2 f$, respectively, the relative standard deviation (RSD) of $S^{2}$ is

$$
R S D\left(s^{2}\right) \equiv \frac{\sqrt{\operatorname{Var}\left(s^{2}\right)}}{E\left(s^{2}\right)}=\sqrt{\frac{2}{f}} .
$$

Therefore,

$$
f=\frac{2}{\left[R S D\left(s^{2}\right)\right]^{2}} \approx \frac{2\left(s^{2}\right)^{2}}{e^{2}} .
$$

(a) This e is a measure of the uncertainty in $\mathrm{s}^{2}$, i.e., an "uncertainty of the uncertainty." 
This approximate $f$ can be used with $s^{2}$ and the method of Section B.6 to combine the external uncertainty estimate with uncertainty estimates derived from HLW vitrification prócess data

To update an uncertainty estimate, prior information must be combined with information contained in a current data set. Combining prior and current information is one application of the branch of statistics known as Bayesian statistics. The second method of updating an external uncertainty estimate utilizes a Bayesian approach. Assume that $n$ current observations, $X_{i}, i=1, \ldots, n$, are available, where the $X_{i}$ are a random sample from a normal distribution with mean zero and variance $\sigma^{2}$. As above, assume that both a prior estimate of $\sigma^{2}$, denoted $\mathrm{s}^{2}$, and a prior estimate of the standard deviation of $\sigma^{2}$, denoted e, are available. Define

$$
\begin{gathered}
\gamma \equiv \frac{\left(s^{2}\right)^{2}}{e^{2}}+2=\frac{s^{4}}{e^{2}}+2 \\
\delta \equiv s^{2}\left(\frac{s^{4}}{e^{2}}+1\right) .
\end{gathered}
$$

Based on these definitions, an updated estimate of $\sigma^{2}$ (one incorporating both the data and the prior information) can be constructed from one possible Bayes estimator:

$$
s_{u}^{2}=\frac{\delta_{u}}{\gamma_{u}-1}
$$

where

$$
\delta_{u}=\delta+\frac{1}{2} \sum_{i=1}^{n} x_{i}^{2}
$$


and

$$
\gamma_{u}=\gamma+\frac{n}{2}
$$

(in each case, the subscript " $u$ " is used to denote an updated estimate). In addition, an updated estimate of the standard deviation of $\sigma^{2}$ can be constructed:

$$
e_{u}=\frac{\delta_{u}}{\left(\gamma_{u}-1\right) \sqrt{\gamma_{u}-2}}=\frac{s_{u}^{2}}{\sqrt{\gamma_{u}-2}}
$$

More discussion of this Bayesian updating method, including derivation of the updated estimators in Equations (B.31) and (B.34), appears in Section 9 and the Appendix of Bryan, Piepel, and Simpson (1994). A multivariate version of this updating scheme appears in Anderson (1984, p. 272).

\section{B.8 REFERENCES}

Anderson, T.W. 1984. An Introduction to Multivariate Analysis, second edition. John Wiley and Sons, New York.

Bryan, M.F. and G.F. Piepel. 1993. Strategy for Product Composition Control in the Hanford Waste Vitrification Plant, PHTD-C93-05.01F, Rev. 0, Pacific Northwest Laboratory, Richland, Washington.

Bryar, M.F. and G.F. Piepel. 1994. Preliminary Feed Test Algorithm for the Hanford Waste Vitrification Plant Product Composition Control System, PHTD-C93-05.01A, Rev. 0, Pacific Northwest Laboratory, Richland, Washington.

Bryan, M.F., G.F. Piepel, and D.B. Simpson. 1994. Methods for Estimation of Covariance Matrices and Covariance Components for the Hanford Waste Vitrification Plant Process, PHTD-C93-05.01P, Rev. 0, Pacific Northwest Laboratory, Richland, Washington.

Gaylor, D.W., and F.N. Hopper. 1969. "Estimating the Degrees of Freedom for Linear Combinations of Mean Squares by Satterthwaite's Formula," Technometrics, 11:691-706. 
Graybill, F.A. 1976. Theory and Application of the Linear Model. Duxbury Press, North Scituate, Rhode Island.

Hrma, P.R., G.F. Piepel, M.J. Schweiger, D.E. Smith, D.-S. Kim, P.E. Redgate, J.D. Vienna, C.A. LoPresti, D.B. Simpson, D.K. Peeler, and M.H. Langowski. 1994. Property/Composition Relationships for Hanford High-Level Waste Glasses Melting at $1150^{\circ} \mathrm{C}$, PVTD-95.02.01B, Pacific Northwest Laboratory, Richland, Washington.

Lindgren, B.W. 1976. Statistical Theory, third edition. MacMillan Publishing Co., Inc., New York.

Satterthwaite, F.E. 1946. "An Approximate Distribution of Estimates of Variance Components," Biometrics Bulletin, 2:110-114.

Searle, S.R. 1971. Linear Models. John Wiley and Sons, New York.

Searle, S.R., G. Casella, and C.E. McCulloch. 1992. Variance Components. John Wiley and Sons, Inc., New York.

Snedecor, G.W., and W.G. Cochran. 1980. Statistical Methods, seventh edition. Iowa State University Press, Ames, Iowa. 


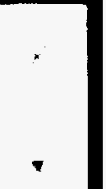

